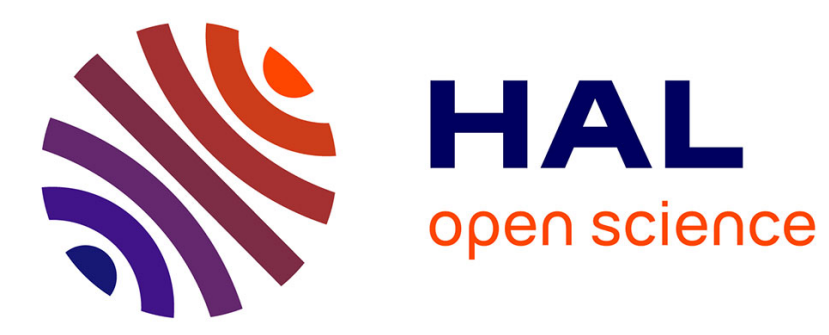

\title{
Effect of interface contamination on particle-bubble collision
}

\author{
Zhujun Huang, Dominique Legendre, Pascal Guiraud
}

\section{To cite this version:}

Zhujun Huang, Dominique Legendre, Pascal Guiraud. Effect of interface contamination on particle-bubble collision. Chemical Engineering Science, 2012, vol. 68 (1), pp. 1-18. 10.1016/j.ces.2011.07.045 . hal-00921117

\section{HAL Id: hal-00921117 https://hal.science/hal-00921117}

Submitted on 19 Dec 2013

HAL is a multi-disciplinary open access archive for the deposit and dissemination of scientific research documents, whether they are published or not. The documents may come from teaching and research institutions in France or abroad, or from public or private research centers.
L'archive ouverte pluridisciplinaire HAL, est destinée au dépôt et à la diffusion de documents scientifiques de niveau recherche, publiés ou non, émanant des établissements d'enseignement et de recherche français ou étrangers, des laboratoires publics ou privés. 


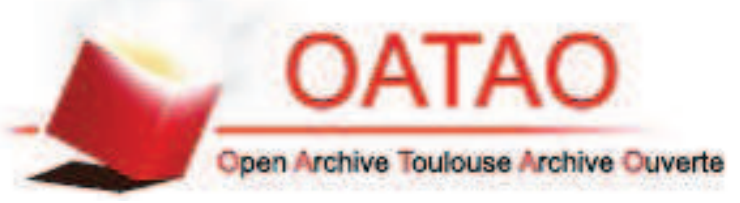

OATAO is an open access repository that collects the work of Toulouse researchers and makes it freely available over the web where possible.

This is an author-deposited version published in : http://oatao.univ-toulouse.fr/ Eprints ID : 10530

To link to this article : DOI: $10.1016 /$ j.ces.2011.07.045

http://dx.doi.org/10.1016/j.ces.2011.07.045

To cite this version : Huang, Zhujun and Legendre, Dominique and Guiraud, Pascal Effect of interface contamination on particle-bubble collision. (2012)

Chemical Engineering Science, vol. 68 (n¹). pp. 1-18. ISSN 0009-2509

Any correspondance concerning this service should be sent to the repository administrator: staff-oatao@listes-diff.inp-toulouse.fr 


\title{
Effect of interface contamination on particle-bubble collision
}

\author{
Z. Huang a,b,c,d,e ${ }^{\text {D. D. Legendre }}{ }^{\text {d,e }}$, P. Guiraud ${ }^{\text {a,b,c,* } *}$ \\ a Université de Toulouse; INSA, UPS, INP; LISBP, 135 Avenue de Rangueil, F-31077 Toulouse, France \\ ${ }^{\mathrm{b}}$ INRA, UMRA792 Ingénierie des Systèmes Biologiques et des Procédés, F-31400 Toulouse, France \\ c CNRS, UMR5504, F-31400 Toulouse, France \\ ¿ Université de Toulouse; INPT, UPS; IMFT (Institut de Mécanique des Fluides de Toulouse), Allée Camille Soula, F-31400 Toulouse, France \\ e CNRS; IMFT; F-31400 Toulouse, France
}

Keywords:

Bubble

Particle

Hydrodynamics

Simulation

Surface contamination

Inertial forces

\begin{abstract}
A B S T R A C T
This study focuses on the impact of the interface contamination on the collision efficiency between bubbles and inertial particles. The bubble's surface mobility has been integrated into the collision modelling by using the hydrodynamics stagnant-cap model, in which the clean angle $\theta_{\text {clean }}$ is used to characterise the interface contamination level. Direct numerical simulations have been performed for various bubble's Reynolds numbers $\left(1 \leq R_{b} \leq 100\right)$, particle to bubble size ratio $\left(0.001 \leq r_{p} / r_{b} \leq 0.02\right)$ and particle's Stokes numbers $\left(0.001<S t_{p}<1\right)$. The Lagrangian tracking was performed for the solid particles by solving the full particle trajectory equation, in order to find the critical grazing trajectory. The collision efficiency was then calculated, as the ratio of the number of particles located in the body of revolution made by critical trajectory to that of particles located in the cylinder formed by bubble's projection area. The magnitude of hydrodynamic force (buoyancy, drag, shear lift, added mass and history forces) as well as surface forces (electrostatic, Van der Waals and hydrophobic forces) are compared to propose a simplified trajectory equation. The surface contamination was found to play an important effect on the behavior of collision efficiency, especially near $\theta_{\text {clean }}$. Analysis of collision angle showed that there is a critical angle $\theta_{\text {crit }}$, depending on the bubble's Reynolds number. For the bubble with $\theta_{\text {clean }}>\theta_{\text {crit }}$, the contact point of the "grazing trajectory" can only be situated on the mobile interface, while for $\theta_{\text {clean }}<\theta_{\text {crit }}$, the contact point may be on both mobile and immobile part of the interface and only the positive inertial effect is observed. A simple model has been proposed that makes possible the description of collision efficiency for clean or contaminated bubbles.
\end{abstract}

\section{Introduction}

Capturing small particles in suspension by micro-bubbles is a widely used method in chemical industry, like water treatment, mineral separation and liquid metal purification. Particle-bubble interaction controls the flotation efficiency during heterocoagulation. It combines particle motion in the fluid displaced by the bubble, the dynamics of collision and the physico-chemical properties of interfacial forces linking the bubble and particles that finally form an aggregate. An overall capture efficiency is usually defined as the ratio between the number of particles captured by a bubble and the number of particles in the volume swept out by this bubble. This heterocoagulation capture efficiency is generally considered as the product of the contributions of three successive steps (Schulze, 1989; Ralston et al., 2002):

\footnotetext{
* Corresponding author at: Université de Toulouse; INSA, UPS, INP; LISBP, 135 Avenue de Rangueil, F-31077 Toulouse, France.

E-mail address: pascal.guiraud@insa-toulouse.fr (P. Guiraud).
}

collision, attachment and particle-bubble aggregate stability. Experimentally, it is not easy to evaluate each contribution, because these three sub-processes are not completely discrete, only the total probability is accessible. However, as the governing forces for each step are independent, the collision process is controlled by the hydrodynamics governing the bubble-particle approach in the liquid phase. If the separation distance is reduced to sub-micrometer order, interfacial forces get involve and the liquid film between the bubble and the particle surface will drain. If the film rupture occurs, the three-phase contact line (boundary layer between the solid surface, liquid phase and gas phase) moves until a stable wetting perimeter is established. Drainage, rupture and contact line movement constitute the attachment process (Nguyen and Schulze, 2004). Detachment may occur if the external forces or kinetic energy exceed the tenacity of the bubble-particle aggregate. The stability of the aggregate is governed by capillary forces. Therefore, they can be treated separately to simplify the modeling of each process. Since only those particles encountering the bubble can be attached to the bubble and be finally separated from fluid, a complete 
comprehension of the elementary collision process before the particle enters the interfacial zone (before contact stage) is the first work to be addressed. According to Schulze (1989), particlebubble collision mechanisms includes interception, gravitational sedimentation, inertial collision, Brownian diffusion, turbulent diffusion and cloud effect. Collision may be dominated by one or several mechanisms, depending on the liquid flow around bubble, particle's weight and density. In most particle-bubble collision studies, it is often supposed that the particle size is very small compared to the bubble size. This assumption leads to the case of collision by interception where particles' inertia is neglected, so the particles trajectory can be simplified to be assimilated to the liquid streamlines. The study of Moruzzi and Reali (2010) shows that in the contact zone of DAF (Dissolved Air Flotation), the distance between bubbles is about 10-20 times bubble diameter, so bubbles can be considered as not interacting. Based on this assumption, models for the collision efficiency have been derived. The first interception collision model can be dated to Sutherland (1948), in which collision efficiency was shown to be a function of the particle to bubble size ratio $E_{\text {coll }}=3 r_{p} / r_{b}$ for potential flow $\left(R e_{b} \rightarrow \infty\right)$. Gaudin (1957) developed the same approach but considering Stokes flow $\left(R e_{b}=0\right)$ around a solid sphere (i.e. a bubble with a fully contaminated surface) and found $E_{\text {coll }}=$ $\frac{3}{2}\left(r_{p} / r_{b}\right)^{2}$. Analytical solutions for intermediate flow were lately given by Yoon and Luttrell (1989), Heindel and Bloom (1999), Nguyen and Kmet (1992). Thanks to an approximation of the flow fields near bubble surface by using Taylor expansion, Weber (1981), Weber and Paddock (1983), Nguyen $(1994,1998)$ have successively proposed other efficiency expressions, where the effect of gravitational sedimentation was considered. Inertial effect on particle-bubble collision was examined by using analytical derivation (Flint and Howerth, 1971) for Stokes flow and potential flow, and then by using numerical simulations (Dobby and Finch, 1987) for intermediate bubble Reynolds numbers. In their works, inertial effect was expressed through the particle's Stokes number $S t=2 \rho_{p} U_{b} r_{p}^{2} / 9 \mu_{f} r_{b}$. The collision efficiency was shown to be significantly increased by particles inertia when $S t>1$. Dukhin et al. (1995) revealed the negative inertial effect, shown as a "centrifugal force" on collision efficiency, which is related to the tangential component of fluid velocity at the interface. Nevertheless, these calculations were based on the assumption of completely contaminated interface. Ralston et al. (1999, 2002) proposed a model involving particle inertia effects without considering gravity for potential flow, i.e. around clean bubbles in the limit of large Reynolds numbers. In these works, the probability of second collision due to rebound of the inertial particle has also been discussed. Plate (1989) tried to evaluate the overall collision efficiency by combining the inertial, gravitational and interception collision efficiencies. However, the overall collision efficiency is not always a simple addition of these effects. Especially, when the collision efficiency is controlled by two or more effects, a complex relationship is generally derived to predict the behavior of the collision process (Schulze, 1989). Nguyen (1998) used Taylor expansion to solve liquid flow around bubble up to $R e_{b}=500$ and obtain a complete model where the inertial and gravity effects on the trajectories of solid particles are included. More recently, in Phan et al. (2003) and Nguyen and Nguyen (2009), the Basset-Boussinesq-Oseen equation for particles is solved for a clean and fully contaminated bubble by taking into consideration the effect of particle density.

The works cited above have considered the collision efficiency with major attention having been paid to two extreme situations: bubbles with clean surface or fully contaminated bubbles. However, it is now well known that not only the surface of fresh bubble is mobile, but the forward surface of aged bubbles where bubble-particle capture takes place can also be mobile (Dukhin et al., 1995; Sam and Gomez, 1996). In practical applications, bubbles are contaminated due to surfactants, contaminants, impurities and/or captured particles. Surfactant adsorbed at the bubble interface or captured particles migrate along the interface to the rear stagnation point, because of surrounding liquid motion or the gravitational effect in the latter case. Depending on their concentration and the adsorption capacity of the interface, the bubble can be totally or partially contaminated. The distribution of contaminant depends on the tangential advection, the diffusion along the interface, the adsorption and desorption at interface (Stone, 1990). When the tangential advection dominates the diffusion, surfactants accumulate at the rear part of the bubble, while the forward surface of bubbles is still mobile (Cuenot et al., 1997). The bubble interface can be divided into two regions and a sharp transition exists between the clean and the stagnant areas. In such condition the bubble surface can be described by using the stagnant cap model. The existence of stagnant cap was first confirmed experimentally by Savic (1953). The corresponding analytical solution in Stokes flow was obtained by Sadhal and Johnson (1983) and several studies have computationally or analytically studied the effects of surfactant on bubbles motion for low to moderate Reynolds numbers (McLaughlin, 1996; Cuenot et al., 1997). Comparisons between the measured and the calculated rise velocity given by Bel Fdhila and Duineveld (1996) and Alves et al. (2005) also confirmed that the stagnant cap assumption reasonably describe this phenomena. The induced effect of a partially contaminated interface on the capture efficiency was considered numerically by Sarrot et al. (2005). It was clearly shown that the flow field around a bubble, strongly influenced by the level of contamination, plays a significant effect on the behavior of the probability of collision: a linear or quadratic dependency of $E_{\text {coll }}$ in $r_{p} / r_{b}$ is found depending on the interface contamination level. Based on numerical simulations and hydrodynamical arguments, Legendre et al. (2009) give a modeling of the effect of the partial contamination of the bubble interface. For a partially contaminated bubble, collision probability behaviors are given by the flux of particles near the surface which is controlled by the tangential velocity for mobile interfaces and by the velocity gradient for immobile interfaces. The influence of the rear stagnant cap on the attachment and detachment have been analysed in particular by Mishchuk et al. (2001). Moreover, as it has been noted by Zholkovskij et al. (2000), the formation of the stagnant cap is a time dependent process. The characteristic angle varies within the floating time as well as the bubble's rise velocity. As a result the collision efficiency may be different along the flotation column. These studies on partially contaminated bubble help us to model correctly the behavior of a whole flotation column. However, these studies were limited to the inertial free particles. When inertia is considered, its induced effect may be different from the mobile to the immobile interface, and the collision probability behavior as result maybe also changed. In this paper, we focus on the collision aspect between a partially contaminated spherical bubble and inertial particles in suspension, with the emphasis on the effects of the particle inertia and its gravitational sedimentation on the collision probability for different bubble surface contamination levels.

\section{Statement of problem}

Appropriate prediction of flotation efficiency during heterocoagulation is the key to modelling of a flotation cell, which requires establishing a kinetic model to describe the number of particles collected per unit of time. If the flotation tank is considered as a Continuous Ideally Stirred-Tank Reactor (CISTR), 
for a first-order reaction, the temporal variation of the particle concentration can be written as

$Q C_{0}-Q C-k C V=0$

So we have

$C(t)=\frac{C_{0}}{1+k \tau}$

where $k$, the flotation rate constant, is defined as the product of the number of bubbles $n_{b}$ and the collection efficiency of each bubble $k=n_{b} \times E_{\text {capt }}$.

If the spatial variation of particle concentration is considered, like as that in a flotation column, the variation of the particle concentration in a finite volume $d V=\pi D^{2} d z / 4$ equals to

$d C(z)=-d n_{p} / d V$

The number of particles captured in each volume $d V$ equals to

$d n_{p}=n_{b} \times E_{\text {capt }} \times C(z) \times \pi r_{b}^{2} d z$

where $n_{b}$ the number of bubbles in $d V$ is related to the gas fraction $\Phi: n_{b}=3 \Phi d V / 4 \pi r_{b}^{3}$. If we suppose that the collection efficiency is constant along the flotation column, integration of Eq. (3) over the column height $L$ gives

$C(z)=C_{0} \exp \left(-\frac{3 L \Phi E_{\text {capt }}}{4 r_{b}}\right)$

And the flotation rate constant can be written as

$k=+\frac{3 Q_{g} L E_{\text {capt }}}{2 d_{b} V}$

So we can see that whatever the type of flotation cell is, the determination of the flotation rate constant requires knowledge of the capture efficiency $E_{\text {capt }}$ of each bubble, which is generally considered as the product of efficiencies of collision $\left(E_{\text {coll }}\right)$, attachment $\left(E_{\text {att }}\right)$ and stabilisation $\left(1-E_{\text {det }}\right)$. It should be noted that in reality the collection efficiency is not always constant and experimental data showed that $E_{\text {capt }}$ decreases along the flotation column (Huang et al., 2011). Particles captured by a bubble modify the latter's surface contamination level and so its collision efficiency (Legendre et al., 2009).

To well understand the effect of the the interfacial contamination on the collection efficiency, we consider here a spherical bubble of radius $r_{b}$ rising straightly at its terminal velocity $U_{b}$ in a liquid at rest of infinite extent containing a uniform suspension of small spherical particles settling at the velocity $U_{s}$. The problem is solved in the frame of reference moving with the bubbles as shown in Fig. 1. We consider a non-deformable bubble which is an acceptable assumption for air-water system for sub-millimetric diameter. The objective of this study is to determine the efficiency of the particle-bubble collision before contact stage. If the concentration of particles is uniform in the liquid, the collision efficiency $E_{\text {coll }}$ can be calculated as the ratio of the flux $Q_{c}$ of particles which collide with the bubble surface and the flux

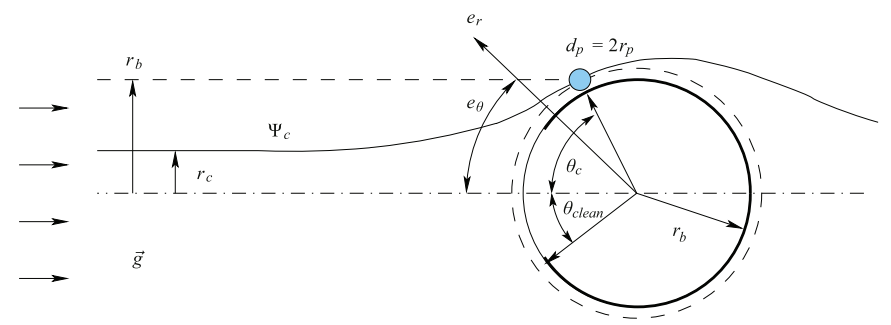

Fig. 1. Schematic view of particle-bubble collision. passing through a cylinder of section $r_{b}^{2}$ :

$E_{\text {coll }}=\frac{Q_{c}}{\pi r_{b}^{2} U_{b}}$

$Q_{c}$ is determined by searching the "grazing trajectory" $\Psi_{c}$ that separates the trajectories of particles that encounter the bubble from those that do not (Schulze, 1989). Only the particles located in the body of revolution made by these grazing trajectories can encounter the bubble. So $Q_{c}$ corresponds to the flow rate passing through the cross section area of radius $r_{c}$ limited by the grazing trajectories upstream far from the bubble: $Q_{c}=r_{c}^{2} U_{b}$. The collision efficiency $E_{\text {coll }}$ can be then written as follows:

$E_{\text {coll }}=\left(\frac{r_{c}}{r_{b}}\right)^{2}$

The numerical calculation of $E_{\text {coll }}$ requires the flow field generated by the bubble and the induced trajectory of the particles. When a particle moves along the grazing trajectory $\Psi_{c}$, it collides the surface at the angle $\theta_{c}$, called collision angle.

The objective is to consider intermediate level of interface contamination on the collision efficiency. As shown in Fig. 1, the bubble contamination or recovering by particles is described using the stagnant cap model (Sadhal and Johnson, 1983), where the bubble surface contamination is characterized via the angle $\theta_{\text {clean }}$ limiting contaminated and clean areas. The forward part of bubble surface $\left(\theta<\theta_{\text {clean }}\right)$ is free of contaminants or particles and moves with the liquid (mobile surface) while the backward bubble surface $\left(\theta>\theta_{\text {clean }}\right)$ is covered by contaminants or particles and behaves as a "stagnant cap" (immobile surface).

\subsection{Governing equations}

The fluid is assumed to be Newtonian and incompressible. We denote its local velocity and pressure by $\mathbf{U}$ and $P$, respectively. The fluid motion around the bubble is obtained by solving the NavierStokes equations:

$\nabla \cdot \mathbf{U}=0, \quad \rho_{f}\left(\frac{D \mathbf{U}}{D t}+\mathbf{U} \cdot \nabla \mathbf{U}\right)=\nabla P+\mu_{f} \Delta \mathbf{U}$

where $\rho_{f}$ and $\mu_{f}$ are the density and the viscosity of the fluid, respectively.

This study is limited to axi-symmetrical flows around the bubble. For a fully contaminated bubble (i.e. solid sphere), the wake loses its axi-symmetry at $R e_{b}=210$ where two vortex filaments appear. For a clean spherical bubble, no vortex appears and the wake is steady and axi-symmetric even at large Reynolds number. Path instability and vortex shedding can appears behind a spherical bubble due to the contamination of bubble surface because vorticity production is increased. The transition from axi-symmetric to non-axi-symmetric wake for a partially contaminated bubble has not yet been identified, it is therefore difficult to clearly indicates the limit of validity of the axisymmetric simulations reported in this study. As we will show, the collision occurs on the front part of the bubble even for partially contaminated bubble so the wake destabilization is not expected to have a significant impact on the results presented here. Finally, the flow displacement generated by the bubble is controlled by two non-dimensional numbers, the bubble rising Reynolds number $\operatorname{Re}_{b}=2 r_{b} \rho_{f} U_{b} / \mu_{f}$ and the level of contamination characterized by $\theta_{\text {clean }}$.

\subsection{Particle motion}

A particle moving in a fluid experiences several forces: gravity force, buoyancy force, drag force, added inertial mass force, stress gradient force, shear lift force and Basset-Boussinesq history 
force. Usually the equation of Maxey and Riley (1983) is used to describe the particle's trajectory. Whereas, at short separation distance (about sub-micro), the interfacial forces as the electrostatic force, Van der Waals forces and other non-DLVO forces may be involved in the interaction between the particle and the bubble. So a particle trajectory equation can be described as below:

$\frac{d \mathbf{x}}{d t}=\mathbf{V}$

with

$$
\begin{aligned}
\rho_{p} \frac{4 \pi r_{p}^{3}}{3} \frac{d \mathbf{V}}{d t}= & \rho_{p} \frac{4 \pi r_{p}^{3}}{3} \mathbf{g}-\rho_{f} \frac{4 \pi r_{p}^{3}}{3} \mathbf{g} \\
& +C_{D} \rho_{f} \frac{\pi r_{p}^{2}}{2}|\mathbf{U}-\mathbf{V}|(\mathbf{U}-\mathbf{V})+C_{M} \rho_{f} \frac{4 \pi r_{p}^{3}}{3}\left(\left.\frac{D \mathbf{U}}{D t}\right|_{p}-\frac{d \mathbf{V}}{d t}\right) \\
& +\left.\rho_{f} \frac{4 \pi r_{p}^{3}}{3} \frac{D \mathbf{U}}{D t}\right|_{p}+C_{L} \rho_{f} \frac{4 \pi r_{p}^{3}}{3}(\mathbf{U}-\mathbf{V}) \times \mathbf{\Omega} \\
& +6 \pi \mu r_{p} \int_{0}^{t} K(t-s)\left[\frac{\partial(\mathbf{U}-\mathbf{V})}{\partial s}\right] d s+\sum \mathbf{F}_{\text {surf }}
\end{aligned}
$$

where $C_{D}, C_{M}$ et $C_{L}$ are, respectively, the drag coefficient, the added mass coefficient and the lift coefficient, $K=r_{p} / \sqrt{\pi v_{f}(t-s)}$ is the kernel of the Basset-Boussinesq history force. $\sum \mathbf{F}_{\text {surf }}$ is the sum of the surface forces that depend on the particles physicochemical properties, particles size and the separation distance. In this expression, $d / d t$ and $D / D t$ denote the time derivatives taken along the particle path and the continuous phase path, subscript " $p$ " denotes continuous-phase conditions evaluated at the particle location in the absence of the particle. In this study, we consider spherical particles so that $C_{M}=1 / 2$. We use Schiller and Nauman (1935) correlation $C_{D}=24 / R e_{p}\left(1+0.15 R e_{p}^{0.687}\right)$ for the drag coefficient and McLaughlin (1991) correlation $C_{L}=9 J(\varepsilon)$ $\left(\operatorname{Re}_{p} S r\right)^{-1 / 2} \pi^{-1}$ with $S r$ the non-dimensional shear rate and $J(\varepsilon)=2.255 /\left(1+0.2 \operatorname{Re}_{p} / S r\right)^{3 / 2}$ given by Legendre and Magnaudet (1998).

The particle inertia effect is described by using the particle Stokes number $S t_{p}$ and the normalized settling velocity $u_{s}=U_{s} / U_{b}$ of the particles. The Stokes number is defined as the ratio between the particle's relaxation time $\tau_{p}$ and the characteristic time induced by the bubble motion $\tau_{b}=2 r_{b} / U_{b}$. Considering Stokes flow condition for the particle motion, the drag coefficient is $C_{D}=24 / R e_{p}$ that yields:

$\mathbf{U}_{s}=\frac{2\left(\rho_{p}-\rho_{f}\right) r_{p}^{2}}{9 \mu} \mathbf{g}$

$\tau_{p}=\frac{2}{9}\left(\rho_{p}+C_{M} \rho_{f}\right) \frac{r_{p}^{2}}{\mu_{f} r_{b}}$

$S t_{p}=\frac{\tau_{p}}{\tau_{b}}=\frac{2}{9}\left(\rho_{p}+C_{M} \rho_{f}\right) \frac{r_{p}^{2} U_{b}}{\mu_{f} r_{b}}=\frac{1}{18}(2 \hat{\rho}+1)\left(\frac{r_{p}}{r_{b}}\right)^{2} R e_{b}$

Relation (14) shows that $S t_{p}, \hat{\rho}, r_{p} / r_{b}$ and $\operatorname{Re}_{b}$ are linked. In the following, the study will be conducted by independently varying $S t_{p}, r_{p} / r_{b}, u_{s}$ and $R e_{b}$.

According to Eq. (8), the numerical calculation of the collision efficiency $E_{\text {coll }}$ requires the determination of $r_{c}$ deduced from the grazing trajectory. This grazing trajectory is obtained by searching the contact point between the bubble surface and particle trajectories. This point is found by trial-and-error, varying the initial particle position $\left(r_{0}, \theta_{0}\right)$ far away from the bubble $\left(\sim 80 r_{b}\right)$, where the particle trajectories are parallel to the symmetrical axis and not influenced by the bubble's motion. $r_{c}$ is determined by moving this initial position until the difference between the minimal distances between the bubble surface and the particles center is less than the required accuracy.

\subsection{Range of parameters}

The above-mentioned parameters for the collision process vary in a very wide range depending upon its application field where collision is involved. As an illustration of these wide ranges, Fig. 2 presents $S t_{p}$ vs. $u_{s}$ for a $d=1 \mathrm{~mm}$ collecting inclusion (gas bubble, solid particle or liquid droplet) for different applications, such as drinking water treatment, selective mineral extraction, deinking process in paper recycling, as well as air purification or rain drop formation, etc., where the densities and viscosities of the collector, of the continuous phase and of the particles to be captured are totally different (see Table 1). The particle's diameter varies between $1 \mu \mathrm{m}$ and $500 \mu \mathrm{m}$. As extreme values conduce to asymptotical results, the direct numerical simulation and lagrangian tracking of the grazing trajectory have been limited to $1<R e_{b}<100,0^{\circ}<\theta_{\text {clean }}<180^{\circ}$, $10^{-4}<S t_{p}<1$ and $10^{-3}<u_{s}<10^{-1}$.

\subsection{Computational method}

Numerical computations reported below were performed by using the JADIM code described in previous works devoted to bubble and particles dynamics (Magnaudet et al., 1995; Cuenot et al., 1997; Legendre and Magnaudet, 1998; Legendre et al., 2003; Merle et al., 2005; Figueroa-Espinoza et al., 2008). Briefly, the JADIM code solves the three-dimensional unsteady Navier-Stokes equations written in velocity-pressure variables in a general system of orthogonal curvilinear coordinates. The discretisation involves a staggered mesh and the equations are integrated in space using a finite volume method with second order accuracy. All spatial derivatives are approximated using second order centred schemes. The time advancement is realized through a RungeKutta/Crank-Nicolson algorithm which is second order accurate in time, and incompressibility is satisfied at the end of each time step by using an auxiliary potential determined by solving a Poisson equation. The computational domain attached to the bubble is a polar domain $(r, \theta)$. The size of the computational domain is chosen as $L_{\infty}=80 r_{b}$ to avoid the confinement of the boundary which is known to have strong effect at low Reynolds number (Magnaudet et al., 1995; Legendre and Magnaudet, 1998). The size of the first cell $\delta$ above the bubble surface has been set to $\delta / r_{b}=0.0005$ according to numerical tests done for the collision efficiency

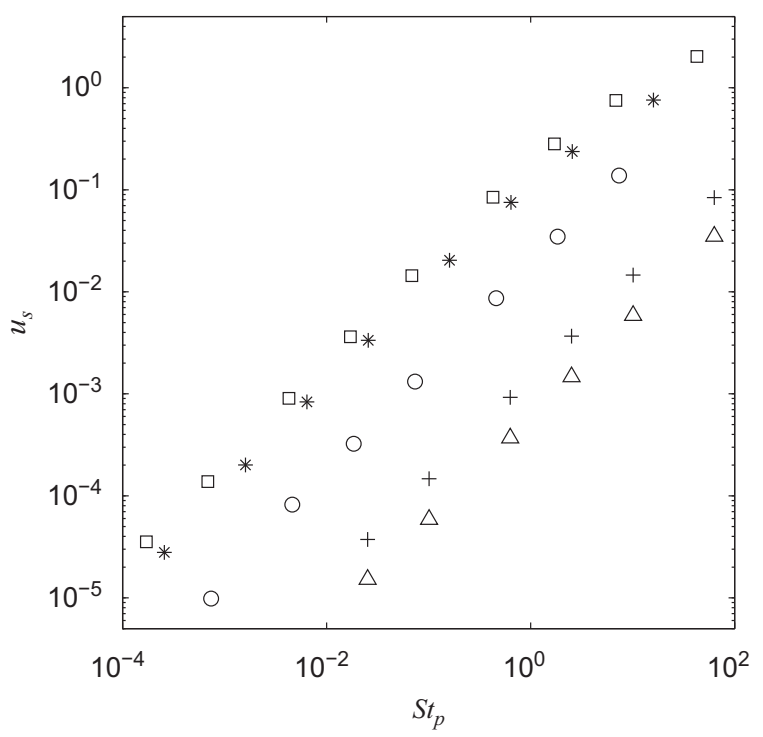

Fig. 2. $S t_{p}$ vs. $u_{s}$ for an inclusion (bubble or drop) $d=1 \mathrm{~mm}$ in different collision applications: Water treatment flotation (*), mineral flotation $(\square)$, deinking process flotation $(\odot)$, air purification $(+)$, rain drop formation $(\triangle)$. Particle size varies from $d_{p}=1 \mu \mathrm{m}$ to $500 \mu \mathrm{m}$. 
Table 1

Physical properties of dispersed and continuous phases in different collision processes.

\begin{tabular}{|c|c|c|c|c|c|c|}
\hline Process & Collector $(b)$ & Particles to be captured $(p)$ & Continuous phase $(f)$ & $\rho_{p} / \rho_{f}$ & $\mu_{f}(\mathrm{~Pa} \mathrm{~s})$ & $\mu_{p}(\mathrm{~Pa} \mathrm{~s})$ \\
\hline Water treatment & Gas & Solid & Liquid & 2.5 & $10^{-3}$ & $\infty$ \\
\hline Mineral extraction & Gas & Solid & Liquid & 7.5 & $10^{-3}$ & $\infty$ \\
\hline Deinking process & Gas & Liquid/solid & Liquid & 1.8 & $18 \times 10^{-3}$ & $9-12$ \\
\hline Air purification & Liquid & Solid & Gas & 2118.6 & $18 \times 10^{-6}$ & $\infty$ \\
\hline Rain drop formation & Liquid & Liquid & Gas & 847.5 & $18 \times 10^{-6}$ & $10^{-3}$ \\
\hline
\end{tabular}
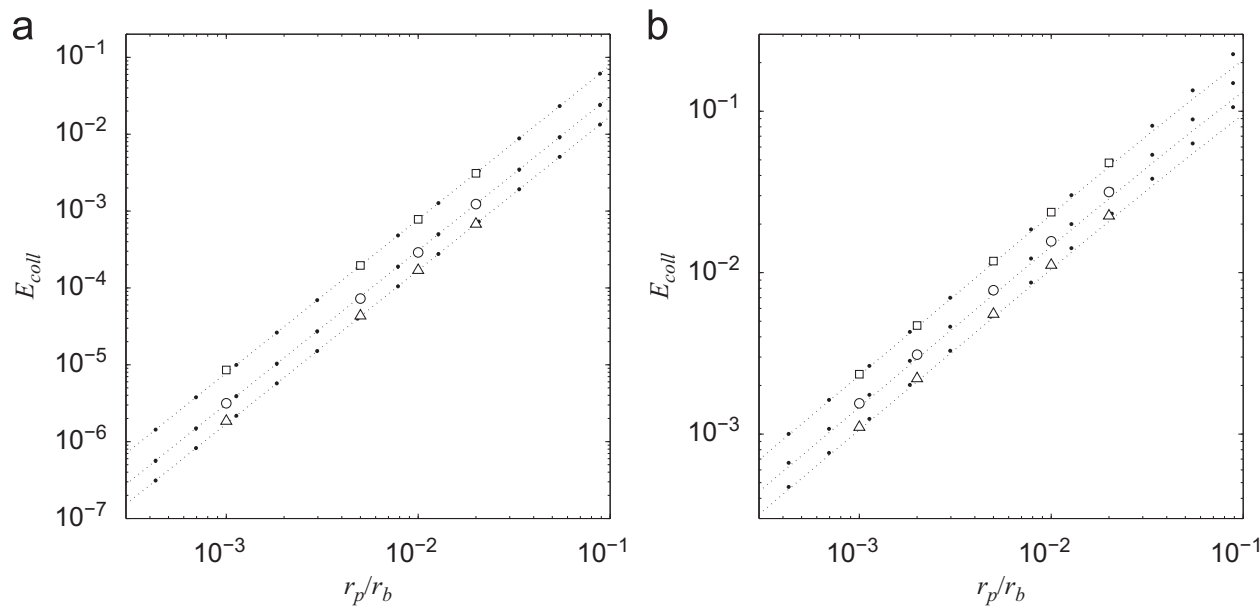

Fig. 3. Collision efficiency $E_{\text {coll }}$ vs. size ratio $r_{p} / r_{b}$ for $S t_{p}=0$ and $u_{s}=0$ at $\operatorname{Re}_{b}=1(\triangle), \operatorname{Re}_{b}=10(\bigcirc)$ and $\operatorname{Re}_{b}=100$ ( $\square$ ), •, Nguyen (1998), $\cdots$, Sarrot et al. (2005). (a) Clean bubble. (b) Fully contaminated bubble.

(Sarrot et al., 2005). In the radial direction, a geometrical progression of nodes ensures that the length ratio between two successive cells is less than 1.1. A constant spacing is used in the $\theta$-direction. Finally, the grid is made of $N_{\theta} \times N_{r}=90 \times 70$ nodes.

The simulation are performed in the reference frame fixed with the bubble. Different boundary conditions are imposed on the computational domain. On the outer boundary, the inflow velocity $-U_{b}$ is imposed upstream $\left(0^{\circ}<\theta<90^{\circ}\right)$ and a parabolic approximation of the governing equation allowing the flow generated by the bubble wake to leave freely the domain without inducing significant perturbations is imposed downstream $\left(90^{\circ}<\theta<180^{\circ}\right)$ :

$\frac{\partial^{2} p}{\partial r \partial \theta}=0, \quad \frac{\partial^{2} U}{\partial r^{2}}=0$

At the bubble surface, the Stagnant Cap Model (Sadhal and Johnson, 1983) is used to model the surface contamination level (Fig. 1). For the mobile part of the bubble surface $\left(0^{\circ}<\theta<\theta_{\text {clean }}\right)$, a zero normal velocity and zero tangential stress condition is imposed:

$\mathbf{U} \cdot \mathbf{n}=0, \quad \mathbf{n} \times(\tau \cdot \mathbf{n})=0$

while for the immobile part $\left(\theta_{\text {clean }}<\theta<180^{\circ}\right)$, a no-slip condition is imposed at the interface:

$\mathbf{U}=0$

Validation of the hydrodynamic simulations for partially contaminated bubble can be found in Sarrot et al. (2005). It has been performed via comparisons with literature results concerning the flow field and the drag coefficient.

Particle trajectory equation is integrated in time using a second order Runge-Kutta method. The fluid velocity and velocity gradients are interpolated at the particle location using a second order accuracy interpolation (Climent and Magnaudet, 1999). The convergence criteria used to determine the value of $r_{c}$ is $10^{-6} r_{b}$ corresponding to $10^{-3}$ time the smaller value of $r_{p}$ considered.

\subsection{Validation for non-inertial particles}

As explained above, when particles inertia induced effects can be neglected $\left(S t_{p} \rightarrow 0, u_{s} \rightarrow 0\right)$, the particles follow the liquid streamlines $(\mathbf{v}=\mathbf{u})$ and the collision occurs by interception. In order to validate our trajectory solver and the numerical parameters used, the system of Eqs. (10) and (11) is considered for $S t_{p}=0$ and $u_{s}=0$, so that the particles trajectories are given by $d \mathbf{x} / d t=\mathbf{V}=\mathbf{U}$. The corresponding values of the collision efficiency v.s. the radius ratio $r_{p} / r_{b}$ are reported in Fig. $3 \mathrm{a}$ and $\mathrm{b}$ at different bubble Reynolds numbers $R e_{b}$ for a perfectly clean bubble and a fully contaminated bubble, respectively. The classical behaviors for both interface conditions are reproduced. For these two situations, at a given value of $r_{p} / r_{b}, E_{\text {coll }}$ increases with $R e_{b}$ as a consequence of the streamlines contraction near the bubble surface. Whatever the Reynolds number $R e_{b}, E_{\text {coll }}$ is found to increase with $r_{p} / r_{b}$. For a fully contaminated bubble, the collision efficiency is a quadratic function of size ratio $E_{\text {coll }} \sim\left(r_{p} / r_{b}\right)^{2}$ while for a clean bubble, the evolution is linear $E_{\text {coll }} \sim r_{p} / r_{b}$. The numerical results were compared with previous results obtained from the value of the stream function (Weber and Paddock, 1983; Nguyen, 1998; Sarrot et al., 2005). All of them give similar prediction. For clarity, the comparison is presented with the model of Nguyen (1998) and numerical results of Sarrot et al. (2005). A good agreement is achieved. The difference with the values deduced from the direct numerical simulations of Sarrot et al. (2005) are less than $2.22 \%$ for a clean bubble and $2.64 \%$ for a totally contaminated bubble.

\section{Particle trajectory analysis}

The objective of this section is to discuss the order of magnitude of the different forces involved is Eq. (11) in order to consider only the dominant effects in the extensive set of simulation that has to be performed. 


\subsection{Drag modification near the interface}

When a particle approaches the bubble surface, its trajectory is influenced by the interaction with the interface (mirror effect). When a particle is transported in the vicinity of a bubble interface with a radius of curvature much larger than the radius of the particle $\left(r_{p} / r_{b} \ll 1\right)$, the interface is seen by the particle as a flat symmetry surface as illustrated in Fig. 4. Solutions for this problem have been proposed in the limit of Stokes flow $\left(R e_{p}=0\right)$ for two particles in interaction (Happel and Brenner, 1965; Kim and Karilla, 1991). Note that in our case, particle's Reynolds number remains very low : $R e_{p} \sim O\left(10^{-7}\right)$.

If the movement of the particle is parallel to the interface, the drag has to be corrected as :

$F=6 \pi \mu r_{p}\left(U_{f}-U_{p}\right) f_{d}^{\perp}$

with

$$
\begin{aligned}
f_{d}^{\perp}= & 1-\frac{3}{4}\left(\frac{r_{p}}{l}\right)+\frac{9}{16}\left(\frac{r_{p}}{l}\right)^{2}-\frac{59}{64}\left(\frac{r_{p}}{l}\right)^{3}+\frac{465}{256}\left(\frac{r_{p}}{l}\right)^{4} \\
& -\frac{15813}{7168}\left(\frac{r_{p}}{l}\right)^{5}+2 \frac{\left(r_{p} / l\right)^{6}}{1+\left(r_{p} / l\right)}
\end{aligned}
$$

where $l$ is the separation distance between the particle and its images.

If the movement of the particle is perpendicular to the interface, the drag is corrected as:

$F=6 \pi \mu r_{p}\left(U_{f}-U_{p}\right) f_{d}^{\|}$

with

$$
\begin{aligned}
f_{d}^{\|}= & 1+\frac{3}{2}\left(\frac{r_{p}}{l}\right)+\frac{9}{4}\left(\frac{r_{p}}{l}\right)^{2}+\frac{19}{8}\left(\frac{r_{p}}{l}\right)^{3}+\frac{93}{16}\left(\frac{r_{p}}{l}\right)^{4}+\frac{387}{32}\left(\frac{r_{p}}{l}\right)^{5}+\frac{1197}{64}\left(\frac{r_{p}}{l}\right)^{6} \\
& +\frac{5331}{128}\left(\frac{r_{p}}{l}\right)^{7}+\frac{19821}{256}\left(\frac{r_{p}}{l}\right)^{8}+\frac{76115}{512}\left(\frac{r_{p}}{l}\right)^{9}+\frac{3}{10} \frac{\left(2 r_{p} / l\right)^{10}}{1+\left(2 r_{p} / l\right)}
\end{aligned}
$$

This correction of the drag force has been included in the trajectory equation in order to determine its influence on the collision efficiency. The correction factors $f_{d}^{\perp}$ and $f_{d}^{\|}$are shown in Fig. 5 as function of the separating distance $h=l-2 r_{p}$ between the particle and the interface. When the particle is close to the bubble at $h / r_{p}=0.01$, the factor $f^{\perp}$ decreases to 0.7 and the factor $f^{\|}$ increases up to 4.5. This parallel motion near the interface may result in a significant increase in drag force.

We have then tested the effect of the drag correction on the collision efficiency for a small particles Stokes number $\left(S t_{p}=0.003\right)$ because it concerns particles whose motion is controlled by the flow generated by the bubble motion. The results are given in

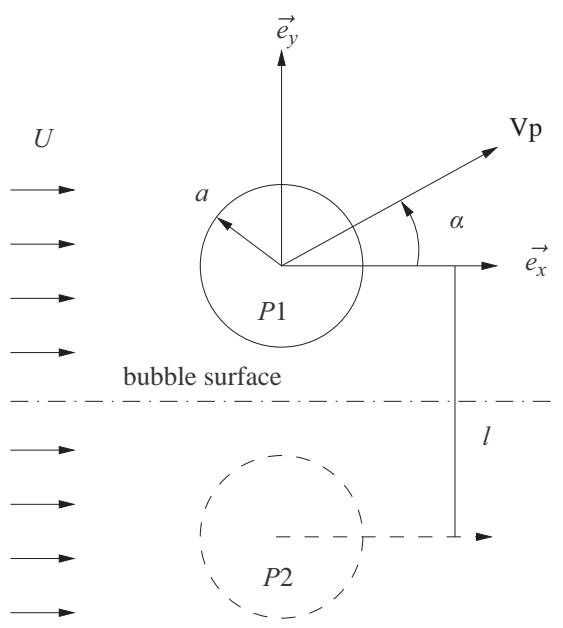

Fig. 4. Schematic of a particle approaching a plane interface.

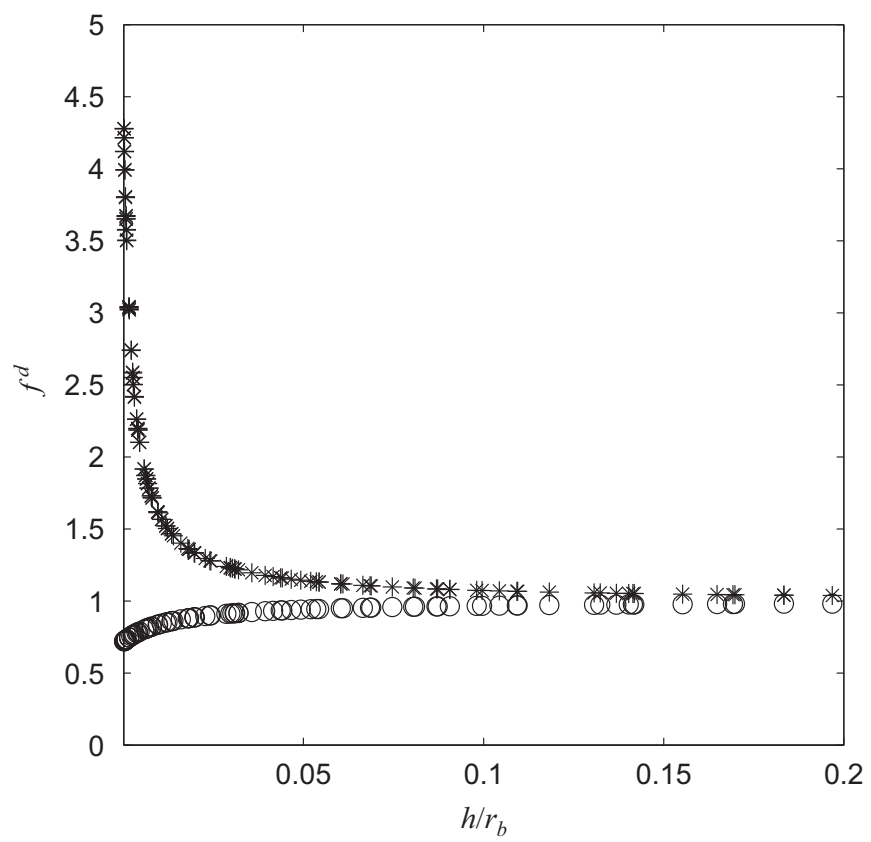

Fig. 5. Evolution of the drag correction of a particle approaching the surface of a bubble. $\circ, f_{d}^{\perp}$ and $*, f_{d}^{\|}$.

Table 2

Effect of with drag correction on collision efficiency ( $S t_{p}=0.003, \rho_{p} / \rho_{f}=2.0$ ).

\begin{tabular}{lllll}
\hline$\theta_{\text {clean }}$ & & $R e_{b}=1$ & $R e_{b}=10$ & $R e_{b}=100$ \\
\hline $180^{\circ}$ & $E_{\text {coll }}$ & $1.075 \times 10^{-2}$ & $1.437 \times 10^{-2}$ & $2.288 \times 10^{-2}$ \\
& $E_{\text {coll }}^{*}$ & $1.027 \times 10^{-2}$ & $1.380 \times 10^{-2}$ & $2.229 \times 10^{-2}$ \\
& $\Delta$ & $4.70 \%$ & $4.12 \%$ & $2.66 \%$ \\
$0^{\circ}$ & $E_{\text {coll }}$ & $1.698 \times 10^{-4}$ & $2.897 \times 10^{-4}$ & $7.808 \times 10^{-4}$ \\
& $E_{\text {coll }}^{*}$ & $1.695 \times 10^{-4}$ & $2.886 \times 10^{-4}$ & $7.783 \times 10^{-4}$ \\
& $\Delta$ & $0.20 \%$ & $0.38 \%$ & $0.31 \%$ \\
\hline
\end{tabular}

Table 2. The critical radius and the collision efficiency for a clean and a fully contaminated bubble with a particle of $r_{p}=0.01 r_{b}$ are calculated. The subscript $(*)$ is for the case with the drag correction. It appears that for a totally contaminated bubble $\left(\theta_{c a p}=0^{\circ}\right)$, the drag correction does not change the collision efficiency $E_{\text {coll }}$ for all concerned $R e_{b}$. Meanwhile, for a clean bubble $\left(\theta_{\text {clean }}=180^{\circ}\right)$, the collision efficiency decreases slightly (2.66-4.70\%).

\subsection{Interfacial force}

As it is shown in Eq. (11), as a particle moving close to a bubble, its trajectory is not only influenced by the hydrodynamic forces, but also by the short distance interfacial forces. After the collision, there is still a thin liquid film that separates the solid particles from the air bubble. The experimental measurement of Yordan and Yoon (1989) has shown that its thickness is about $110 \mathrm{~nm}$. Note that surface forces begin to be involved in the bubble-particle interaction when the separation distance between the surfaces is of the order of magnitude of sub-micron, the collision may be controlled by surface forces at small separation distance. In general, the surface forces to be considered are electrostatic force $F_{R}$, Van der Waals forces $F_{A}$ and hydrophobic forces $F_{H}$. The effects of these three types of force have been tested by using the expression and the constants given in the 
literature (Yoon, 2000; Nguyen et al., 2001; Nguyen and Evans, 2004):

(a) Electrostatic force $F_{R}$ :

$$
F_{R}(h)=\varepsilon \varepsilon_{0} \kappa \frac{2 \pi r_{b} r_{p}}{r_{b}+r_{p}} \frac{2 \zeta_{p} \zeta_{b} \exp (\kappa h)+\zeta_{p}^{2}+\zeta_{b}^{2}}{\exp (2 \kappa h)-1}
$$

where $\varepsilon_{0}$ is the vacuum permittivity, $\varepsilon$ is the relative permittivity of the solution, $\zeta$ is the zeta potential and $\kappa^{-1}$ is the Debye length. The electrostatic charges on the surface are characterized by the zeta potential of the particle and liquid properties. The measures of the zeta potential for an air bubble approaching to a slice surface taken by Yordan and Yoon (1989) show that $\zeta_{b}=-45 \mathrm{mV}$ and $\zeta_{p}=-20 \mathrm{mV}$.

(b) Van der Waals forces $F_{A}$ :

$$
\begin{aligned}
F_{A}(h)= & \frac{A}{6}\left\{-\frac{4 \pi r_{b} r_{p} r_{p b}}{\left[r_{p b}^{2}-\left(r_{p}+r_{b}\right)^{2}\right]^{2}}-\frac{4 \pi r_{b} r_{p} r_{p b}}{\left[r_{p b}^{2}-\left(r_{p}-r_{b}\right)^{2}\right]^{2}}\right. \\
& \left.+\frac{8 \pi r_{b} r_{p} r_{p b}}{\left[r_{p b}^{2}-\left(r_{p}+r_{b}\right)^{2}\right]\left[r_{p b}^{2}-\left(r_{p}+r_{b}\right)^{2}\right]^{2}}\right\}
\end{aligned}
$$

where $r_{p b}$ is the distance between the center of the bubble and that of the particle $r_{p b}=r_{b}+r_{p}+h$, and $A$ is the Hamaker (1937) constant. According to Yordan and Yoon (1989), $A=-3.12 \times 10^{-21}$ for a system slice-air bubble immersed in water.

(c) Hydrophobic forces $F_{H}$ :

$$
F_{H}=\frac{r_{p} r_{b}}{r_{p}+r_{b}}\left[C_{1} \exp \left(-h / \lambda_{1}\right)+C_{2} \exp \left(-h / \lambda_{2}\right)\right]
$$

The coefficients $C 1, C 2, \lambda_{1}$ and $\lambda_{2}$ can be obtained only by AFM (atomic force microscope) measurements. The experimental data of Craig et al. (1999) give $C_{1}=-7 \mathrm{mV} / \mathrm{N}, C_{2}=-6 \mathrm{mV} / \mathrm{N}$, $\lambda_{1}=6 \mathrm{~nm}$ and $\lambda_{2}=20 \mathrm{~nm}$.

It should be noted that all the expressions used are valid only in the condition that the surface charge is uniform. On the contrary, different expressions should be used for the clean and the contaminated surface (Vold, 1961; Usui and Barouch, 1990; Mishchuk, 2005). However, in the first part of the trajectory, the interaction is with the clean part.

In Fig. 6, these surface forces as well as hydrodynamic forces acting on a solid silica particle moving towards an air bubble of $R e_{b}=100$ (corresponding to $d_{b}=0.6 \mathrm{~mm}$ ) in pure water have been shown as function of the separation distance. It is observed that the Van der Waals force $(\nabla)$ is very low in comparison to the others for $h / r_{b}>2 \times 10^{-4}$. Its order of magnitude is less than $10^{-15}$. Note that at very short separation distance $(0-50 \mathrm{~nm})$, Van der Waals forces may be significant, surpass other forces, and change from attractive to repulsive (Mishchuk et al., 2002; Nguyen et al., 2001). Since both the particle and the bubble carry negative charges, the electrostatic interaction $(*)$ is repulsive. Only the hydrophobic force $(\diamond)$ is shown to be attractive. In this stage, it is still the hydrodynamics forces that get the particle approach towards the bubble surface. Meanwhile, as the separation distances $h / r_{b}$ decreases to under $10^{-4}$ (about $100 \mathrm{~nm}$ ), the effect of these interfacial forces becomes significant. At the same time, the drag $(O)$ begins to decrease. This distance corresponds to the thickness of the liquid film (Yoon, 2000). Under this distance, the interfacial forces control the drainage, corresponding to the beginning of the attachment process. Particle and bubble could not get closer furthermore as long as the liquid film is not drained off. Since the objective of our study is focused on the collision step before the contact stage, $h=100 \mathrm{~nm}$ has been defined as the limit of computational domain. However, as the

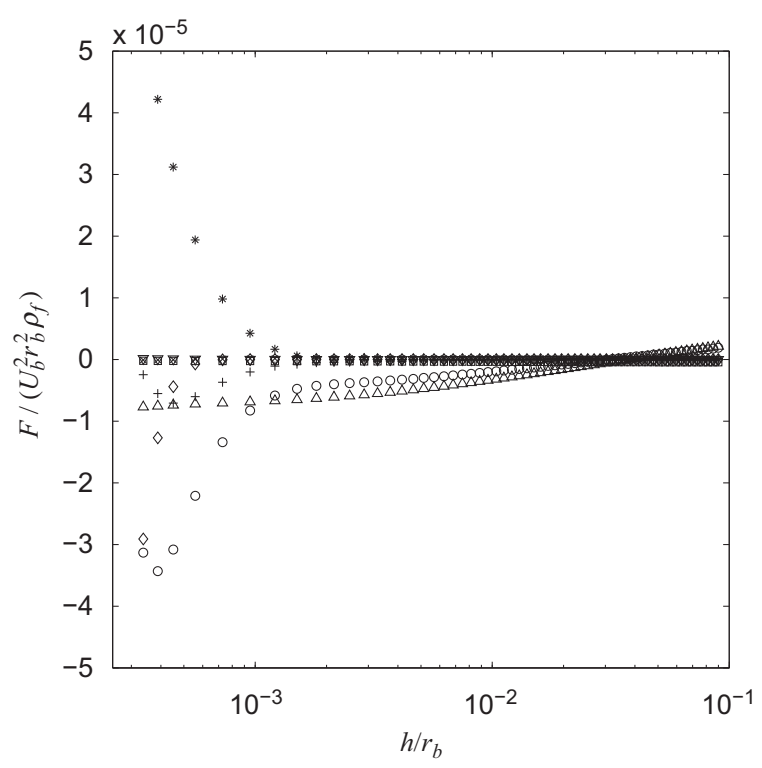

Fig. 6. Comparison of the forces applying to a particle moving close to a clean bubble $\left(R e_{b}=100, r_{p} / r_{b}=0.01, S t_{p}=0.01, \hat{\rho}=2.5\right)$ as function of the separation $h$ between the particle and the surface. $\square$, buoyancy; $\circ$, drag; $\triangle$, added mass; $\times$, shear lift; +, history force; $*$, electrostatic force; $\nabla$, Van der Waals forces; $\diamond$, hydrophobic force.

Table 3

Effect of with interfacial forces on collision efficiency $\left(S t_{p}=0.003, \rho_{p} / \rho_{f}=2.0\right.$ and $\theta_{\text {clean }}=180^{\circ}$ ).

\begin{tabular}{lllll}
\hline$\theta_{\text {clean }}$ & & $R e_{b}=1$ & $R e_{b}=10$ & $R e_{b}=100$ \\
\hline $180^{\circ}$ & $E_{\text {coll }}$ & $1.075 \times 10^{-2}$ & $1.437 \times 10^{-2}$ & $2.288 \times 10^{-2}$ \\
& $E_{\text {coll }}^{*}$ & $1.077 \times 10^{-2}$ & $1.441 \times 10^{-2}$ & $2.375 \times 10^{-2}$ \\
& $\Delta$ & $0.23 \%$ & $0.35 \%$ & $3.70 \%$ \\
\hline
\end{tabular}

interfacial forces begin to be of the same order of magnitude as the hydrodynamic forces at $h / r_{b}=10^{-3}$, it is necessary to examine their influence on the bubble-particle collision. We have then tested the effect of the surface forces on the collision efficiency $E_{\text {coll }}$ for a clean bubble. Table 3 shows that the interfacial forces are favorable for the bubble-particle collision, but the increase of collision efficiency is not significant.

\subsection{Simplified trajectory equation}

Fig. 6 shows also the evolution of the hydrodynamic forces acting on a solid particle along its trajectory $\left(r_{p} / r_{b}=0.01\right.$, $S t_{p}=0.01, \hat{\rho}=2.5$ and $u_{s}=2.72 \times 10^{-2}$ ). The particle Reynolds number $R e_{p}$ is found to evolve between $10^{-4}$ and $10^{-2}$ clearly indicating that the choice of Stokes drag is justified. For larger Stokes numbers, the particle Reynolds number increases and becomes comparable to unity. For this reason the Schiller and Neuman drag force is used in the simulations. The drag force and the added-mass force are shown to be the two forces dominant in the particle-bubble collision. They increase significantly as the particle approaches the bubble surface. Meanwhile, the lift force and the history force are observed to be of a second-order forces and may be neglected as noted by Nguyen (2003). This has been confirmed by our numerical simulation results of the collision efficiency. In Tables 4 and 5, the influence of the lift force and the Basset history force on the collision efficiency are reported, respectively. The subscript $(*)$ represents the case in which the lift force or the history force is accounted. It is noted that either for a clean bubble or for a totally contaminated bubble, the influences of 
Table 4

Effect of accounting lift force on collision efficiency $\left(S t_{p}=0.003, \rho_{p} / \rho_{f}=2.0\right.$ ).

\begin{tabular}{lllll}
\hline$\theta_{\text {clean }}$ & & $R e_{b}=1$ & $R e_{b}=10$ & $\operatorname{Re}_{b}=100$ \\
\hline $180^{\circ}$ & $E_{\text {coll }}$ & $1.075 \times 10^{-2}$ & $1.437 \times 10^{-2}$ & $2.288 \times 10^{-2}$ \\
& $E_{\text {coll }}^{*}$ & $1.039 \times 10^{-2}$ & $1.435 \times 10^{-2}$ & $2.283 \times 10^{-2}$ \\
& $\Delta$ & $3.37 \%$ & $0.10 \%$ & $0.20 \%$ \\
$0^{\circ}$ & $E_{\text {coll }}$ & $1.698 \times 10^{-4}$ & $2.897 \times 10^{-4}$ & $7.808 \times 10^{-4}$ \\
& $E_{\text {coll }}^{*}$ & $1.695 \times 10^{-4}$ & $2.921 \times 10^{-4}$ & $7.879 \times 10^{-4}$ \\
& $\Delta$ & $0.15 \%$ & $0.82 \%$ & $0.92 \%$ \\
& & &
\end{tabular}

Table 5

Effect of accounting history force on collision efficiency $\left(S t_{p}=0.003, \rho_{p} / \rho_{f}=2.0\right.$ ).

\begin{tabular}{lllll}
\hline$\theta_{\text {clean }}$ & & $R e_{b}=1$ & $R e_{b}=10$ & $R e_{b}=100$ \\
\hline $180^{\circ}$ & $E_{\text {coll }}$ & $1.075 \times 10^{-2}$ & $1.437 \times 10^{-2}$ & $2.288 \times 10^{-2}$ \\
& $E_{\text {coll }}^{*}$ & $1.039 \times 10^{-2}$ & $1.437 \times 10^{-2}$ & $2.291 \times 10^{-2}$ \\
& $\Delta$ & $3.37 \%$ & $0.02 \%$ & $0.16 \%$ \\
$0^{\circ}$ & $E_{\text {coll }}$ & $1.698 \times 10^{-4}$ & $2.897 \times 10^{-4}$ & $7.808 \times 10^{-4}$ \\
& $E_{\text {coll }}^{*}$ & $1.695 \times 10^{-4}$ & $2.887 \times 10^{-4}$ & $7.784 \times 10^{-4}$ \\
& $\Delta$ & $0.15 \%$ & $0.35 \%$ & $0.29 \%$ \\
\hline
\end{tabular}

these two forces are limited to $3.4 \%$ relative, so they can be safely neglected in the full particle trajectory equation (Eq. (11)) for intensive simulation.

To conclude, we have shown that the dominant forces are the drag, inertia and buoyancy. History and lift force play a second role and can be neglected. The effect of the drag modification close to the interface on the collision efficiency value remains limited $(<4.7 \%)$. Interfacial forces take advantage on hydrodynamic forces at a short distance from the bubble surface $(\sim 100 \mathrm{~nm})$. Accounting these physico-chemical forces has little effect on collision efficiency $(<3.7 \%)$. Moreover, both drag modification and interfacial forces effects remain very small in comparison with the severe impact of interface contamination that can modify the collision efficiency by several orders of magnitude (Sarrot et al., 2005). So finally, the trajectory equation can be simplified in order to only take into consideration the dominant effects: drag, inertia and buoyancy. The simplified dimensionless form of Eq. (11) controlling the particle motion before the attachment can be written as:

$S t_{p} \frac{d \mathbf{v}}{d \tau}-S t_{p}\left(\frac{3}{2 \hat{\rho}+1}\right) \frac{D \mathbf{u}}{D \tau}=(\mathbf{u}-\mathbf{v})+\mathbf{u}_{\mathbf{s}}$

with $\tau=U_{b} t / r_{b}, \hat{\rho}=\rho_{p} / \rho_{f}, \mathbf{u}=U / U_{b}, \mathbf{v}=\mathbf{V} / U_{b}$ and $\mathbf{u}_{\mathbf{s}}=\mathbf{U}_{\mathbf{s}} / U_{b}$. In Eq. (25), the first and second terms on the left hand side characterize the particle's inertia and the liquid inertial force (including added mass with $C_{M}=1 / 2$ ), respectively. For non-inertial particles (St $\rightarrow 0$ and $u_{s} \rightarrow 0$ ), Eq. (10) reduces to $\mathbf{v}=\mathbf{u}$ and the particles follow the streamlines generated by the bubble motion. This is the general assumption used for inertia free particle bubble collision. The effect of interface contamination on collision efficiency in this simplified case has been reported by Legendre et al. (2009).

\section{Results and discussions}

Direct numerical simulations (DNS) have been performed for various bubble's Reynolds numbers and particle's Stokes numbers. The Navier-Stokes equations has been first solved for the flow field around the bubble. The Lagrangian tracking was performed for the solid particles by solving Eq. (25). Grazing trajectories have been obtained by searching the contact point and the minimal distance $r_{c}$ between the bubble surface and the particles center. The collision efficiency was then calculated, according to its definition (Eq. (8)).

DNS results of the case of a bubble with clean or a fully contaminated surface is first presented, followed by the comparison with the similarly studies existing in the literature. Second, a partially contaminated bubble is considered via the study of the influence of level of interface contamination. Finally, a simple model for estimation of the collision efficiency is proposed.

\subsection{Preliminary results: clean or fully contaminated bubble}

We consider in this section two extreme cases: bubble with a mobile or a completely immobile surface. As we have discussed in Section 2.3, for different applications, the physical properties of the particles vary in a very large range. So here, the effect of the particle's inertia $\left(S t_{p}\right)$ and that of the gravitational sedimentation $\left(u_{s}\right)$ on collision efficiency are discussed separately. The effect of the Stokes number is at first presented in Section 4.1 .1 by imposing $g=0$ in Eq. (25), so that $u_{s}=0$. The effect of the settling velocity is then considered in Section 4.1.2 and finally the results are compared with correlations available in literature in Section 4.1.3.

\subsubsection{Inertial effects for non-settling particles $\left(S t_{p} \neq 0\right.$ and $\left.u_{s}=0\right)$}

Inertial forces have been neglected in many studies that provide models valid for very small particles. The inertial forces have been generally overlooked for medium size particles and bubbles with high velocity (Yoon and Luttrell, 1989; Dobby and Finch, 1987). By taking into consideration the effect of bubble surface mobility, some studies (Dai et al., 1998; Nguyen, 1999) have shown a negative influence of fluid inertia on particle-bubble collision for a mobile bubble surface that induces a decrease of the collision efficiency with inertia. In this section, we first examine the global influence of inertia on particle-bubble collision by varying the particle Stokes number $S t_{p}$, the bubble Reynolds number $R e_{b}$ and the particle to bubble radius ratio $r_{p} / r_{b}$, but neglecting the settling effect $u_{s}=0$. The evolutions of the collision efficiency $E_{\text {coll }}$ and the collision angle $\theta_{c}$ vs. the Stokes number $S t_{p}$ are plotted in Figs. 7 and 8 for a clean and a fully contaminated bubble, respectively, for $R e_{b}=100$ and $0.001 \leq r_{p} / r_{b} \leq 0.02$. As $S t_{p} \rightarrow 0, E_{\text {coll }}$ tends to the value for the inertial-free particle-bubble collision $E_{\text {coll }}^{0}$ (see Fig 3, symbol $\square$ ). Meanwhile, as Stokes number increases from zero, we observe a different behavior between mobile and immobile interface.

Fig. 7 shows that for all the size ratio considered, collision efficiency $E_{\text {coll }}$ of a clean bubble starts to decrease with $r_{p} / r_{b}$. Moreover, $E_{\text {coll }}$ experiences a significant reduction $(\sim 60 \%$ of its value for $S t_{p}=0$ ) until a critical value $S t_{p}^{\text {th }} \approx 0.05$ is achieved. The same behavior has been observed for $R e_{b}=1$ and $R e_{b}=10$ (not represented here). However, for a fully contaminated bubble, $E_{\text {coll }}$ behavior is very different as shown in Fig. 8: the collision efficiency is nearly constant until $S t_{p}^{\text {th }} \approx 0.02$. For larger Stokes number $S t_{p}>S t_{p}^{\text {th }}$, the same behavior is observed for both clean and contaminated bubbles: $E_{\text {coll }}$ grows rapidly with $S t_{p}$ and the dependence on the size ratio $r_{p} / r_{b}$ is not perceptible.

The evolution of the collision angle $\theta_{c}$ is similar for clean and contaminated bubbles: $\theta_{c}$ starts to decrease until its minimum and then increases. For both two cases, $\theta_{c} \approx 68-70^{\circ}$ at $S t_{p}=1$. For a clean bubble, the first regime is also characterized by a significant effect of the radius ratio until $S t_{p}^{\text {th }} \approx 0.05$, where the minimum value is reached. For larger $S t_{p}, \theta_{c}$ is independent on the radius ratio. For a contaminated bubble, the influence of $r_{p} / r_{b}$ on the collision angle $\theta_{c}$ is very small compared to the variations induced by the Stokes number. $\theta_{c}$ reaches its minimum $\theta_{c} \approx$ $25-30^{\circ}$ for $S t_{p}^{\text {th }} \approx 0.3$. 

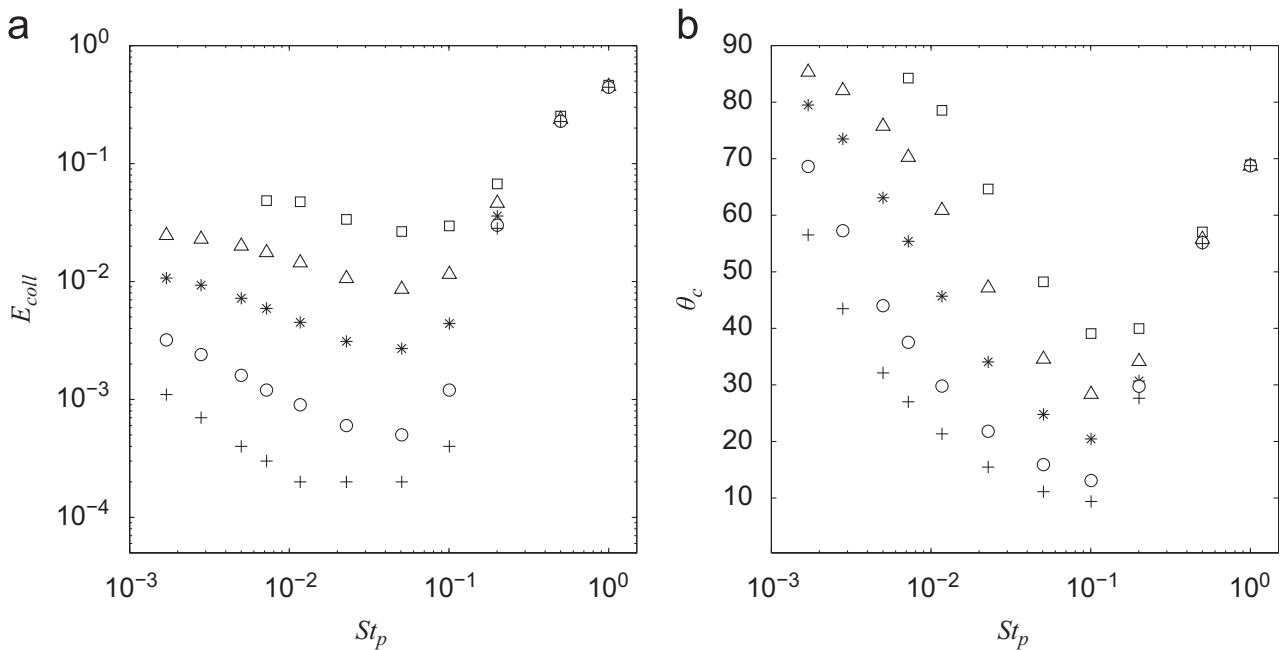

Fig. 7. Collision efficiency $\left(E_{\text {coll }}\right)$ and collision angle $\left(\theta_{c}\right)$ vs. Stokes number $\left(S t_{p}\right)$ for a clean bubble at $\operatorname{Re}_{b}=100$ for non-settling particles $\left(u_{s}=0\right)$. $\square$, $r_{p} / r_{b}=0.02 ; \Delta$, $r_{p} / r_{b}=0.01 ; *, r_{p} / r_{b}=0.005 ; \circ, r_{p} / r_{b}=0.002:+, r_{p} / r_{b}=0.001$. (a) Collision efficiency. (b) Collision angle.
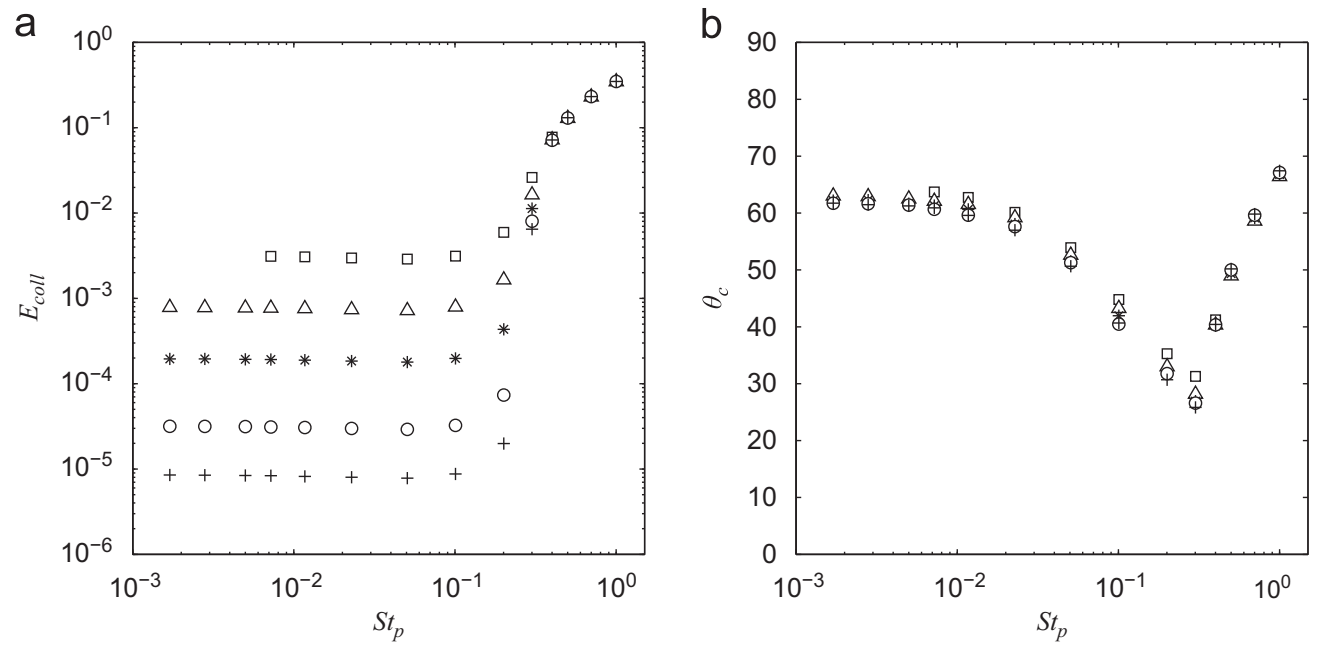

Fig. 8. Collision efficiency $\left(E_{\text {coll }}\right)$ and collision angle $\left(\theta_{c}\right)$ vs. Stokes number $\left(S t_{p}\right)$ for a fully contaminated bubble at $R e_{b}=100$ for non-settling particles $\left(u_{s}=0\right)$. $\square$, $r_{p} / r_{b}=0.02 ; \triangle, r_{p} / r_{b}=0.01 ; *, r_{p} / r_{b}=0.005 ; \circ, r_{p} / r_{b}=0.002 ;+, r_{p} / r_{b}=0.001$. (a) Collision efficiency. (b) Collision angle.

The behavior reported in these figures reveals two opposite contributions induced by inertia (Schulze, 1989; Nguyen, 1994; Dukhin et al., 1995; Dai et al., 2000). As a particle approaches the bubble surface towards the front part of the bubble $\left(\theta<45^{\circ}\right)$, the particle tends to move straightly towards the interface rather than follow the streamlines because of its inertia (see Fig. 9a). This phenomena, called in the following as "positive inertial effect" as introduced by Ralston et al. (1999), tends to increase the probability of collision. As $\theta$ increases to $90^{\circ}$, the radial component of the fluid velocity $U_{r} \sim U_{b} \cos \theta$ (which forces the particles to move towards the bubble) decreases to zero while the tangential component of the fluid velocity $U_{\theta} \sim U_{b} \sin \theta$ increases, so a "centrifugal force" becomes significant near the bubble's equator $\left(45^{\circ}<\theta<90^{\circ}\right)$. This centrifugal effect induced by the fluid inertia pulls particles away from the interface. The consequence is a reduction of the probability of collision and this effect is called "negative inertial effect" (see Fig. 9b). Consequently, if the contact point of the grazing trajectory is located on the front part of the bubble, the positive inertia contribution is dominant and the increase of $S t_{p}$ increases the collision efficiency; if it is located near the equator, the negative inertia effect is dominant and the collision efficiency is decreased. a

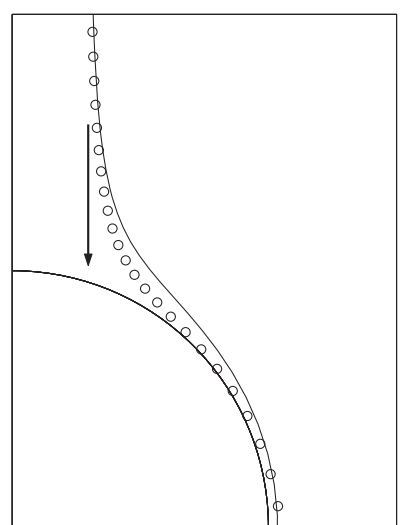

b

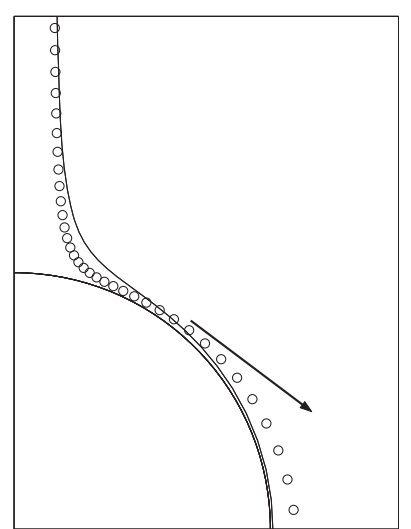

Fig. 9. Inertial effect on particle-bubble collision. (a) Positive effect. (b) Negative effect.

4.1.2. Effect of the gravitational force $\left(S t_{p} \neq 0\right.$ and $\left.u_{s} \neq 0\right)$

When the particle inertia increases, not only the Stokes number effect becomes significant but also gravitational effect 

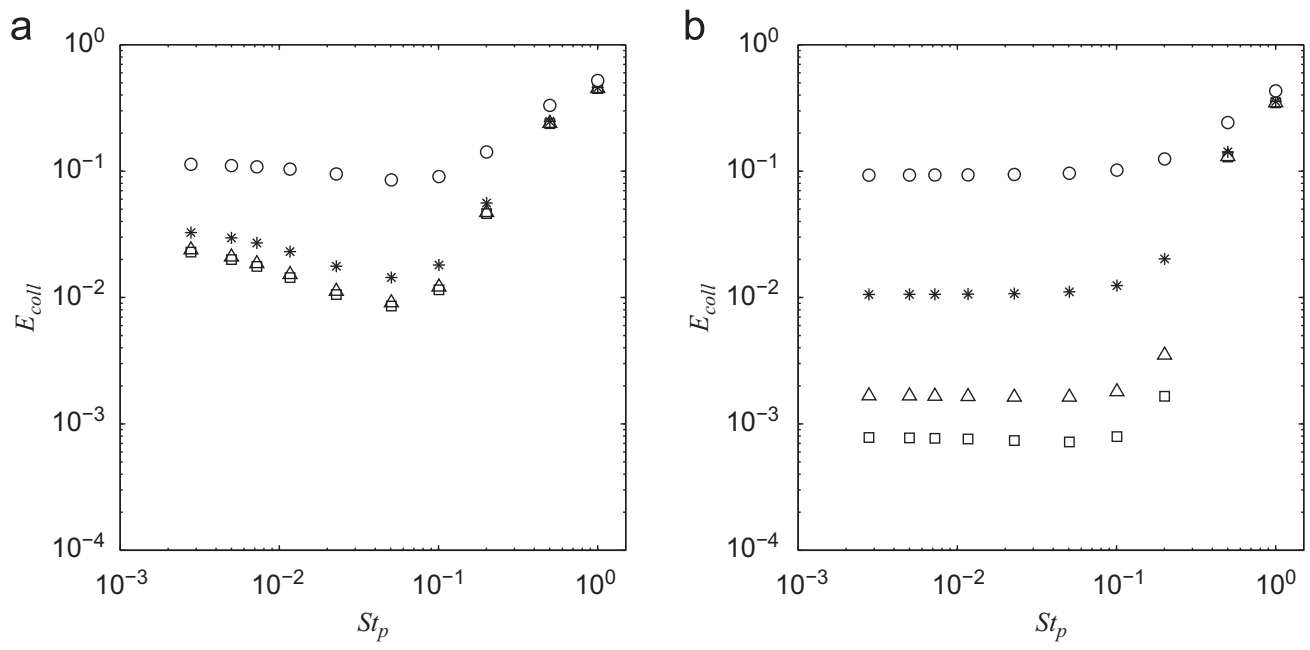

Fig. 10. Gravitational effect for a clean bubble with $r_{p} / r_{b}=0.01$ and $R_{b}=100$. $\square, u_{s}=0 ; \triangle, u_{s}=10^{-3} ; *, u_{s}=10^{-2} ; \circ, u_{s}=10^{-1}$. (a) Clean bubble. (b) Fully contaminated bubble.

has to be considered. The trajectory is now solved by considering these two effects. In order to analyze the effect of the gravitational force, the evolution of the collision efficiency $E_{\text {coll }}$ is plotted at different values of the settling velocity $u_{s}$ in Fig. 10, by varying the gravity $g$ in Eq. (12).

For a clean bubble (Fig. 10a), there is no significant effect of the gravitational force, if $u_{s}$ is smaller than 0.001. In the case of larger values of $u_{s}$, the gravitational sedimentation in favor of particlebubble collision progressively balances the negative effects induces by inertia till $S t_{p} \approx S t_{p}^{\text {th }}$. For $u_{s} \sim 0.1$, these two opposite effects become comparable. The same evolution has been observed for all the Reynolds numbers $R_{b}$ and particle to bubble size ratio $r_{p} / r_{b}$ considered.

For fully contaminated bubbles (Fig. 10b), whatever the value of $u_{s}$, the evolution of $E_{\text {capt }}$ is the same: $E_{\text {coll }}$ remains constant for $S t_{p} \leq 0.1$ and increases for larger values of Stokes number. Difference of $E_{\text {coll }}$ for each $u_{s}$ is reduced as $S t_{p}$ increases and finally $E_{\text {coll }}$ depends only on particles Stokes number. Moreover, when $u_{s}$ increases from 0.001 to $0.1, E_{\text {coll }}$ is increased by two orders of magnitude that is more important than the increase observed for a bubble with clean surface. This difference due to the surface condition can be explained by the fact that a particle takes more time to move around a contaminated surface than a clean surface, since both the fluid and the particles velocities are largely reduced near an immobile interface. As a result, particles have more time to settle down instead of being transported by the fluid so the effect of $u_{s}$ is more important.

The evolution of collision efficiency $E_{\text {coll }}$ of the particles with different Stokes number $S t_{p}$ is plotted in Fig. 11 for a clean bubble at $R e_{b}=100$. The numerical solution for inertial-free particles based on the calculation of streamline function obtained by Sarrot et al. (2005) is also presented in solid line for comparison. It can be seen that at low Stokes number $\left(S t_{p} \leq 0.002\right)$, the collision efficiency is very close to the value obtained for inertia-free particles and evolves as $r_{p} / r_{b}$ ( $S t_{p}=0$, presented by symbols “ $\triangleleft$ ”). For a given size ratio $r_{p} / r_{b}$, the growth of $S t_{p}$ results in an increase of the collision efficiency. The gravitational effect is more significant for small particles than for bigger ones at the same value of $S t_{p}$. Moreover, when $S t_{p}$ exceeds 0.1, $E_{\text {coll }}$ becomes independent on $r_{p} / r_{b}$. In such situation, collision is totally dominated by gravitational sedimentation.

\subsubsection{Discussion}

In this section, the numerical results presented above are compared with models from the literature. The Sutherland (1948)

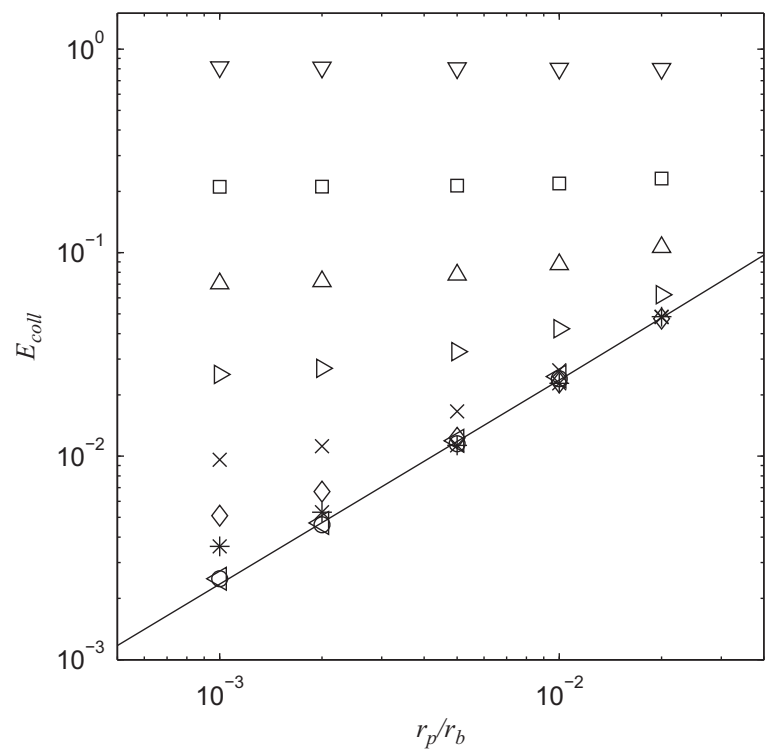

Fig. 11. Collision efficiencies vs. diameter ratio at $R e_{b}=100$ for a fully mobile surface. - , Sarrot et al. (2005); $\triangleleft, S t_{p}=0 ; \circ, S t_{p}=0.002 ; *, S t_{p}=0.005 ; \diamond, S t_{p}=$ $0.01 ; \times, S t_{p}=0.02 ; \triangle, S t_{p}=0.1 ; \square, S t_{p}=0.2 ;+, S t_{p}=0.5 ; \nabla, S t_{p}=1.0$.

model $E_{\text {coll }}^{0}=3 r_{p} / r_{b}$, valid for clean bubbles in the limit of large bubble Reynolds number has been extended to account for the fluid inertia (Dukhin, 1983; Dai et al., 1998), but the settling effect being neglected $\left(u_{s}=0\right)$. The resulting model also referred as the Generalized Sutherland Equation (GSE) writes:

$\frac{E_{\text {coll }}}{E_{\text {coll }}^{0}}=\sin ^{2} \theta_{c} \exp \left[3 K_{D} \cos \theta_{c}\left(\ln \frac{3}{E_{\text {coll }}^{0}}-1.8\right)-\frac{3 K_{D}\left(2+\cos ^{3} \theta_{c}-3 \cos \theta_{c}\right)}{2 E_{\text {coll }}^{0} \sin ^{2} \theta_{c}}\right]$

with

$\theta_{c}=\arccos \left(\sqrt{1+\beta^{2}}-\beta\right), \quad K_{D}=\frac{2 U_{b} r_{p}^{2}\left(\rho_{p}-\rho_{f}\right)}{9 \mu_{f} r_{b}}, \quad \beta=\frac{2 E_{\text {coll }}^{0} f_{d}}{9 K_{D}}$

where $f_{d}$ is a particle drag correction due to the vicinity of the bubble interface. It was considered by Dukhin et al. (1995) to be $f_{d}=2$ in the GSE model. The influence of the drag correction $f_{d}$ on the collision efficiency has been tested and we have observed that this effect has a small contribution (less than $4.7 \%$ ). Based on this 
model, Ralston et al. (2002) proposed a refined GSE for the collision efficiency, in which pressing hydrodynamic force, centrifugal force and short range hydrodynamic interaction have been taken into consideration:

$$
\begin{aligned}
\frac{E_{\text {coll }}}{E_{\text {coll }}^{0}}= & \sin ^{2} \theta_{c} \exp \left[3 K_{D} \cos \theta_{c}\left(1-E_{\text {coll }}^{0}\right)\left(\ln \frac{3}{E_{\text {coll }}^{0}}-1.8\right)\right] \\
& \times\left\{1-\frac{9 K_{D}}{2 E_{\text {coll }}^{0} \sin ^{2} \theta_{c}}\left[p\left(\theta_{c}\right)-4 m \cot \theta_{c} g\left(\theta_{c}\right)\right]\right\}^{\left(1-E_{\text {coll }}^{0}\right)}
\end{aligned}
$$

with

$p\left(\theta_{c}\right)=\int_{0}^{\theta_{c}} \sin ^{5} \theta d \theta, \quad g\left(\theta_{c}\right)=\int_{0}^{\theta_{c}} d \theta \int_{0}^{\theta} p(\theta) d \theta, \quad m=\frac{3 K_{D}}{2} \cos \theta_{c}$

The collision efficiency calculated by Eqs. (26) and (27) and its dependence on $S t_{p}$ are both plotted in Fig. 12. The GSE model (Eq. (26)) correctly reproduces the negative effect of the inertial forces for particles with small Stokes number $\left(S t_{p}<0.1\right)$. An important discrepancy can be observed for particles with high Stokes number, as confirmed by the numerical simulation of Nguyen et al. (2006). Meanwhile, the refined GSE model (Eq. (27)) yields a larger values than that given by the original GSE model (Eq. (26)), since the hydrodynamic pressing force is taken into account. It suppresses the manifestation of the centrifugal force. However, in our study, the calculation is performed out of the interfacial boundary layer before the contact stage. This explains that the simulation results is closer to that given by the original GSE model. Besides that, this model is valid in the limit $R e_{b} \rightarrow \infty$ and consider only the variation of particle's Stokes number. Consequently, an overestimation of the collision efficiency for small $R e_{b}$ is observed, which is not shown here. However, this model has been established for ultra fine particles (typically $d_{p}<10 \mu \mathrm{m}$ ), for this reason, the gravitational forces have not been included.

Numerical solutions are now compared with the model proposed by Nguyen et al. (1998), in which the combined effect of gravity and interception has been taken into consideration. In this

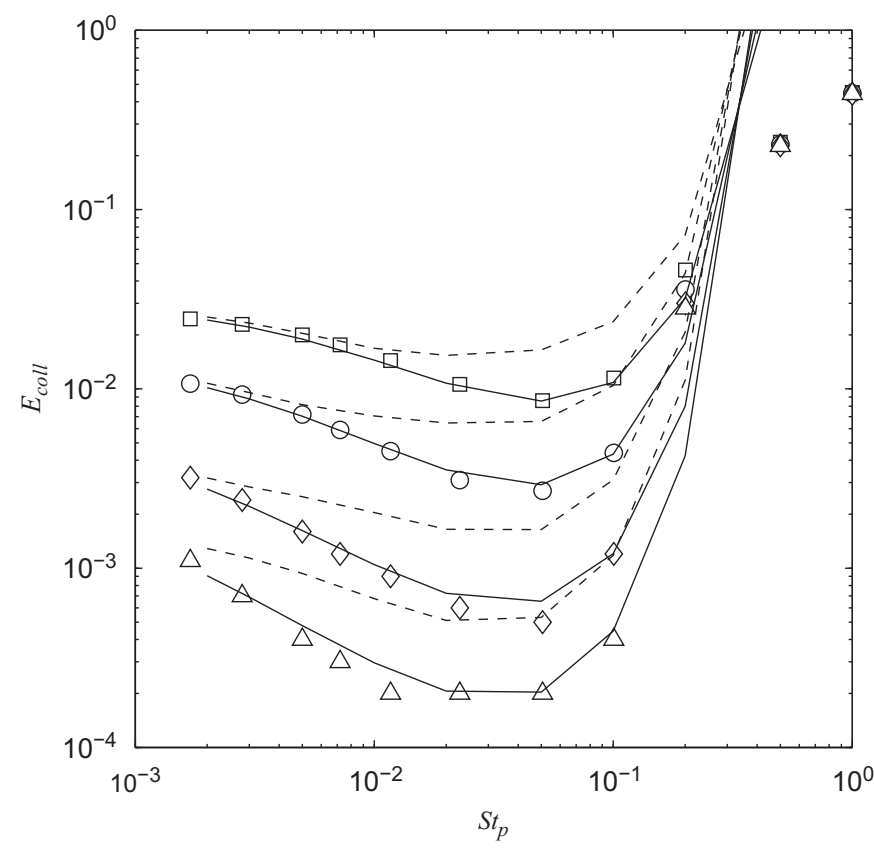

Fig. 12. Comparison with the GSE models (Dai et al., 1998; Ralston et al., 2002) for a clean bubble $R e_{b}=100, u_{s}=0$. DNS results: $\square, r_{p} / r_{b}=0.01 ; \circ, r_{p} / r_{b}=0.005 ; \diamond$, $r_{p} / r_{b}=0.002 ; \triangle, r_{p} / r_{b}=0.001$. GSE model: - Eq. (26), refined GSE model: - - Eq. (27). model, the collision angle is written as:

$\cos \theta_{c}=\frac{\sqrt{\left(X+C_{1}\right)^{2}+C_{2}^{2} X^{4}}-\left(X+C_{1}\right)}{C_{2} X^{2}}$

and the collision efficiency for a totally contaminated bubble is

$E_{\text {coll }}^{\text {im }}=f_{1} \frac{X+C_{1}+Y \cos \theta_{c}}{1+u_{s}} \sin ^{2} \theta_{c}+O\left(S t^{3 / 2}\right)$

with

$X=\frac{3}{2}+\frac{9 R e_{b} / 32}{1+0.309 R e_{b}^{0.694}} \quad$ and $\quad Y=\frac{3 R e_{b} / 8}{1+0.217 R e_{b}^{0.518}}$

while that for a clean bubble it writes as

$E_{\text {coll }}^{m}=f_{1} \frac{\left(X+C_{1}\right) \sin ^{2} \theta_{c}-C_{2} X^{2}\left(\cos ^{3} \theta_{c}-3 \cos \theta_{c}+2\right) / 3}{1+u_{s}}+O\left(S t^{3 / 2}\right)$

with

$X=1+\frac{0.0637 R_{b}}{1+0.0438 \operatorname{Re}_{b}^{0.976}} \quad$ and $\quad Y=\frac{0.0537 R_{b}}{1+0.0318 \operatorname{Re}_{b}^{1.309}}$

In this model $X$ and $Y$ describe the effect of bubble's Reynolds number and depend on the surface mobility. The coefficients $C_{1}$ and $C_{2}$ contain the effect of fluid and particle inertia and are given by

$C_{1}=\frac{u_{s}}{f_{1}}, \quad C_{2}=\frac{S t}{2}\left(1-\frac{\rho_{f}}{\rho_{p}}\right) \frac{f_{2}}{f_{1}}$

with

if $\theta_{\text {clean }}=\pi, \quad f_{1}^{m}=\frac{r_{p}}{r_{b}}-\left(\frac{r_{p}}{r_{b}}\right)^{2} \quad$ and $f_{2}^{m}=1$

if $\theta_{\text {clean }}=0, \quad f_{1}^{\text {im }}=\left(\frac{r_{p}}{r_{b}}\right)^{2}$ and $f_{2}^{\text {im }}=4\left(\frac{r_{p}}{r_{b}}\right)^{2}$

It should be noted that in this equation, the Stokes number is defined as $S t=2 \rho_{p} r_{p}^{2} U_{b} / 9 \mu_{f} r_{b}$ where the effect of the added mass is not considered. In order to compare this model to our numerical simulations, here we use $S t_{p}=S t(\hat{\rho}+0.5)$ to characterize the inertial effects. For small Stokes numbers, a good agreement between the model and the numerical results is obtained both for a bubble with a mobile (see Fig. 13a, $\theta_{\text {clean }}=180^{\circ}$ ) and an immobile surface (see Fig. $\left.13 \mathrm{~b}, \theta_{\text {clean }}=0^{\circ}\right)$. However, as $S t_{p}$ increases, a discrepancy between the model and DNS results appears. It is more significant for a clean bubble than for a fully contaminated bubble. It should be noted that this model is an approximate solutions of Eq. (25) based on the assumption that $S t \sim\left(r_{p} / r_{b}\right)^{2}$. So the last term of Eqs. (29) and (31), $O\left(S t^{3 / 2}\right) \sim O\left(r_{p} / r_{b}\right)^{3} \sim O\left(10^{-6}\right)$ may be neglected. But for large Stokes number, like $S t>0.1$, this term becomes important $O\left(S t^{3 / 2}\right) \sim O\left(10^{-2}\right)$ and should not be neglected. That is certainly one reason for the observed discrepancy for $S t_{p}>0.1$. Finally, we can conclude that the model proposed by Nguyen et al. (1998) is in good agreement with our numerical simulations up to $S t_{p}=0.1$ when the bubble interface is immobile, and up to $S t_{p}=0.02$ when the bubble interface is mobile.

Comparison with models of Schulze (1989) has been reported on Fig. 14 for a clean bubble. Based on the numerical simulations of Plate (1989), Schulze proposed an expression for the overall collision efficiency, where $E_{\text {coll }}$ is the sum of that of its three contributions: interception $E_{\text {coll }}^{i}$, gravitational sedimentation $E_{\text {coll }}^{g}$ and the inertial collision $E_{\text {coll }}^{\text {in }}$ :

$E_{\text {coll }}=E_{\text {coll }}^{i}+E_{\text {coll }}^{g}+E_{\text {coll }}^{\text {in }}\left[1-\frac{E_{\text {coll }}^{i}}{\left(1+r_{p} / r_{b}\right)^{2}}\right]$ 

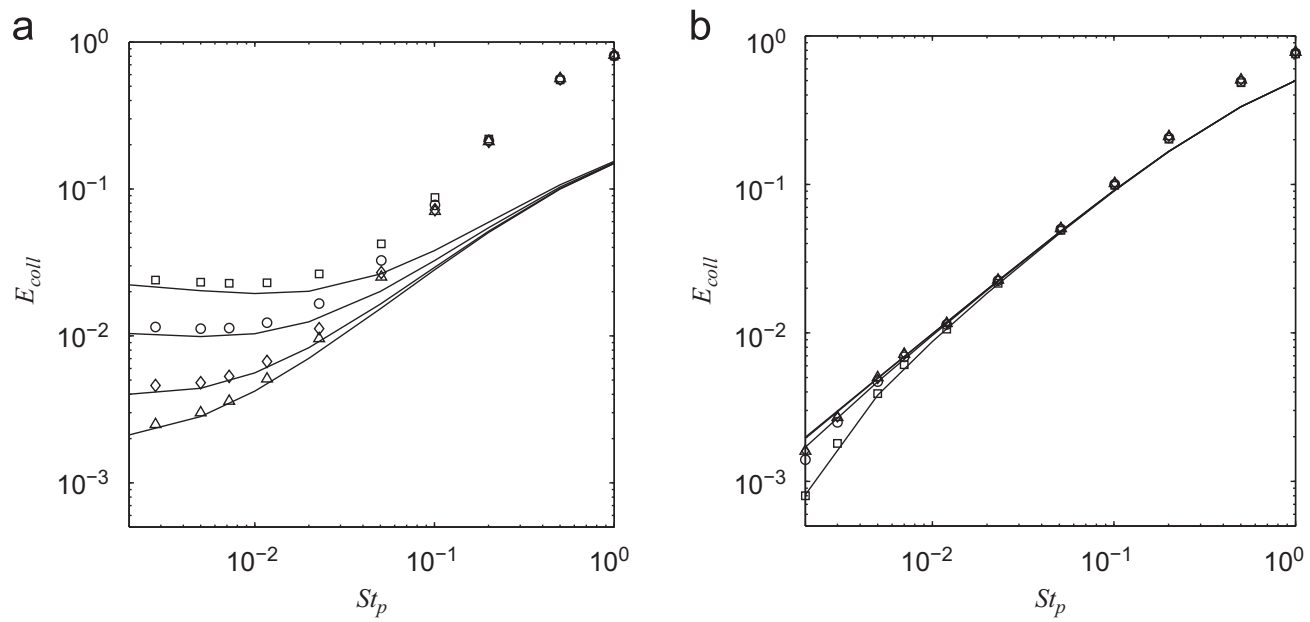

Fig. 13. Comparison with Nguyen et al. (1998) model. - , DNS results for $\operatorname{Re}_{b}=100 ; \square, r_{p} / r_{b}=0.01 ; \circ, r_{p} / r_{b}=0.005 ; \diamond, r_{p} / r_{b}=0.002 ; \triangle, r_{p} / r_{b}=0.001$. (a) Clean bubble. (b) Fully contaminated bubble.
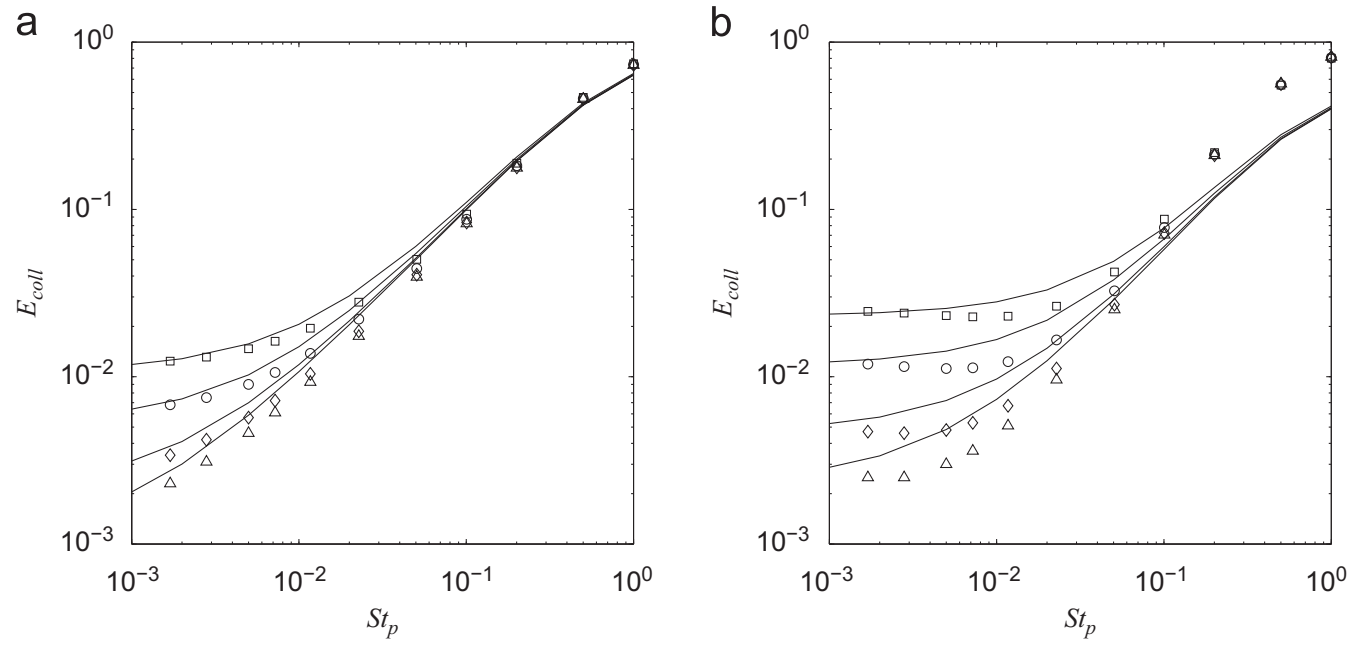

Fig. 14. Comparison with Schulze (1989) model (-). DNS results for a clean bubble at $\operatorname{Re}_{b}=1$ and $100, \square, r_{p} / r_{b}=0.01 ; \circ, r_{p} / r_{b}=0.005 ; \diamond, r_{p} / r_{b}=0.002 ; \triangle, r_{p} / r_{b}=0.001$. (a) Clean bubble at $R e_{b}=1$. (b) Clean bubble at $R e_{b}=100$.

$\left(E_{\text {coll }}^{i}+E_{\text {coll }}^{g}\right)$ can be calculated by the model of Weber (1981), while for $E_{\text {coll }}^{\text {in }}$ Schulze has given an approximate solution:

$E_{\text {coll }}^{\text {in }}=\frac{1}{1+u_{s}}\left(1+\frac{r_{p}}{r_{b}}\right)^{2}\left(\frac{S t}{S t+a}\right)^{b}$

where the constants $a$ and $b$ depend on the bubble's Reynolds number (Plate, 1989). Since $r_{p} / r_{b} \ll 1$, the last term of Eq. (33), which means that only the positive effect of the inertia is considered. This agrees with what is shown in Fig. 14, $E_{\text {coll }}$ increasing monotonously with $S t_{p}$. Notably, Schulze's model well describes the evolution of $E_{\text {coll }}=f\left(S t_{p}\right)$ for small Reynolds number (see Fig. 14a), but as $\operatorname{Re}_{b}$ increases, this model deviates always from the numerical solutions. As we can see in Fig. 14b, for lower Stokes number, it neglects the negative effect of inertia, while it under estimates the positive effect for high Stokes number.

\subsection{Collision with a partially contaminated bubble}

As discussed in Introduction, in practical conditions in flotation, bubble interface can be neither perfectly clean nor fully contaminated. The objective of this section is to consider the effect of a partially contaminated interface on the collision efficiency.
Following the structure of the previous section, we have first investigated this effect for non-settling particles (4.2.1) and then considered all the effect induced by inertia (4.2.2).

4.2.1. Inertial effects for non-settling particles $\left(S t_{p} \neq 0\right.$ and $\left.u_{s}=0\right)$

Figs. 15 and 16 present the collision efficiencies as function of the Stokes number $S t_{p}$ for the bubble Reynolds number $R e_{b}=100$ and $R e_{b}=1$ with a particle to bubble size ratio $r_{p} / r_{b}=0.01$. The gravity is fixed to zero in the trajectory equation, so that $u_{s}=0$. The contamination angle $\theta_{\text {clean }}$ varies from $0^{\circ}$ for a fully contaminated bubble to $180^{\circ}$ for a clean bubble. The strong influence of the surface contamination is clearly put in evidence: the collision efficiency for clean bubbles $E_{\text {coll }}^{m}$ is much greater than that for a fully contaminated bubble $E_{\text {coll }}^{i m}$, which confirms a significant effect of the surface contamination level.

For the bubble with $\theta_{\text {clean }}$ greater than $90^{\circ}$, the point of contact between the particle and the bubble remains on the mobile part of the interface, since the collision always occurs on the forward part of bubble surface. As a result, $E_{\text {coll }}$ behavior is the same as that observed for a clean bubble $E_{\text {coll }}=E_{\text {coll }}^{m}$. Meanwhile, in the case of $\theta_{\text {clean }}$ less than $90^{\circ}, E_{\text {coll }}$ deviates from $E_{\text {coll }}^{m}$ and progressively approaches that for a fully contaminated bubble $E_{\text {coll }}^{i m}$. 

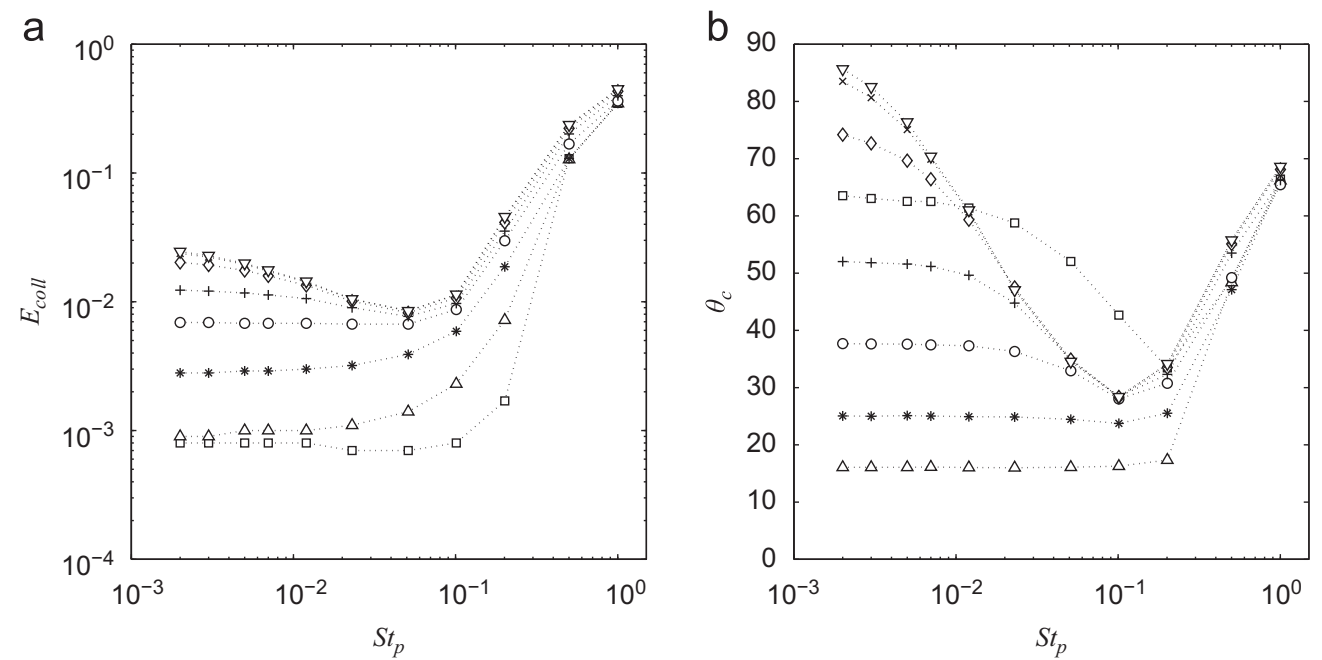

Fig. 15. Collision efficiency $\left(E_{\text {coll }}\right)$ and collision angle $\left(\theta_{c}\right)$ vs. Stokes number $\left(S t_{p}\right)$ for a partially contaminated bubble $\left(r_{p} / r_{b}=0.01\right)$ at $R e_{b}=100$. $\square, \theta_{\text {clean }}=0^{\circ} ; \triangle, \theta_{\text {clean }}=$ $20^{\circ} ; *, \theta_{\text {clean }}=30^{\circ} ; \circ, \theta_{\text {clean }}=45^{\circ} ;+, \theta_{\text {clean }}=60^{\circ} ; \diamond, \theta_{\text {clean }}=90^{\circ} ; \times, \theta_{\text {clean }}=120^{\circ} ; \nabla, \theta_{\text {clean }}=180^{\circ}$. (a) Collision efficiency. (b) Collision angle.
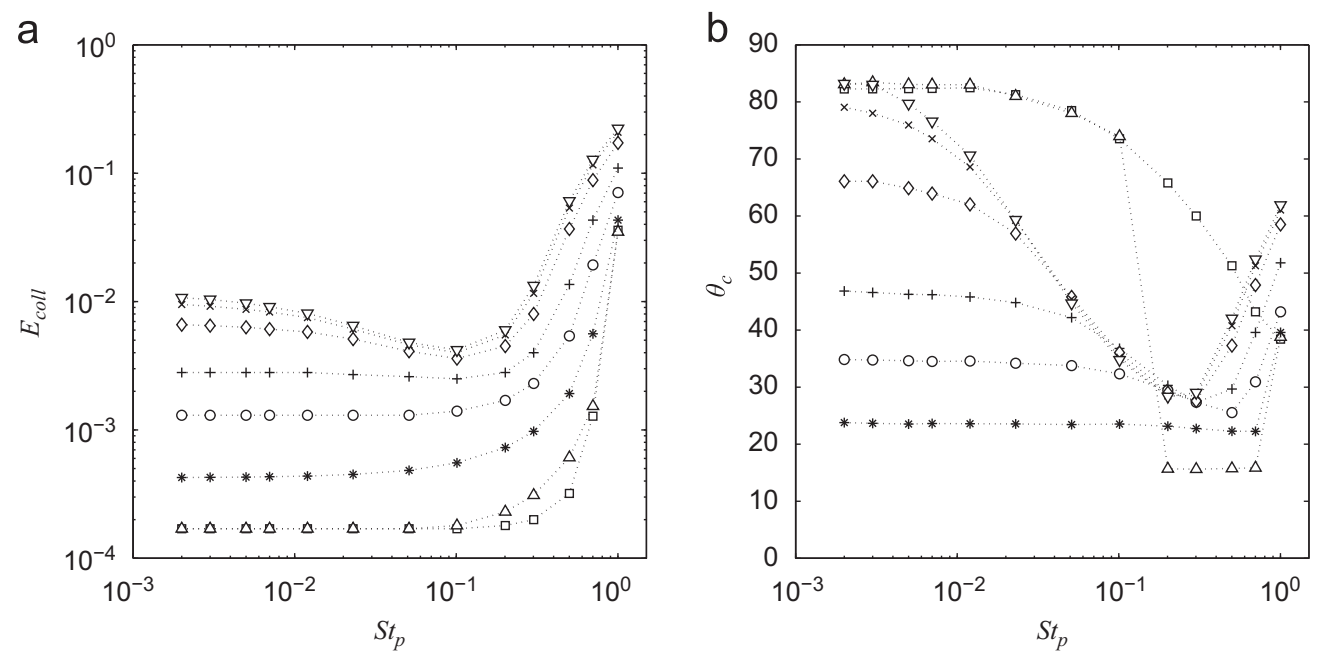

Fig. 16. Collision efficiency $\left(E_{\text {coll }}\right)$ and collision angle $\left(\theta_{c}\right)$ vs. Stokes number $\left(S t_{p}\right)$ for a partially contaminated bubble $\left(r_{p} / r_{b}=0.01\right)$ at $R e_{b}=1$. $\square, \theta_{\text {clean }}=0^{\circ} ; \triangle$, $\theta_{\text {clean }}=20^{\circ}$; $*, \theta_{\text {clean }}=30^{\circ} ; \circ, \theta_{\text {clean }}=45^{\circ} ;+, \theta_{\text {clean }}=60^{\circ} ; \diamond, \theta_{\text {clean }}=90^{\circ} ; \times, \theta_{\text {clean }}=120^{\circ} ; \nabla, \theta_{\text {clean }}=180^{\circ}$. (a) Collision efficiency. (b) Collision angle.

When $\theta_{\text {clean }}$ falls below to $30^{\circ}$, no more decrease of the collision efficiency is observed as $S t_{p}$ increases. Under this conditions, $E_{\text {coll }}$ behavior is the same as that observed for a fully contaminated bubble.

The variation of the collision efficiency as a function of $\theta_{\text {clean }}$ can be explained by the decrease of the tangential velocity of the local fluid near the interface due to the reduction of the surface mobility. Legendre et al. (2009) have calculated and given an approximation of the maximum tangential velocity $U_{\Gamma}$ as a function of $\theta_{\text {clean }}$. When $\theta_{\text {clean }}$ is above $120^{\circ}, U_{\Gamma}\left(\theta_{\text {clean }}\right) \approx U_{\Gamma}\left(90^{\circ}\right)$; meanwhile when $\theta_{\text {clean }}$ is less than $90^{\circ}, U_{\Gamma}$ decreases rapidly with $\theta_{\text {clean }}$ and the negative inertial effect become insignificant. The evolution of collision efficiency in the latter case is close to that of a totally contaminated bubble. However, the positive inertial effect at the front of the bubble always exists for large Stokes number.

Concerning the collision angle $\theta_{c}$, for a given particle to bubble size ratio $r_{p} / r_{b}$, it depends not only on bubble's Reynolds number $R e_{b}$ but also on particle's Stokes number $S t_{p}$ and interface contamination level $\theta_{\text {clean }}$. In the case of small Stokes number $\left(S t_{p}=0.002\right)$, the behavior of $\theta_{c}$ is observed to be the same as that of inertial free particles $\left(S t_{p}=0\right)$ already described by Legendre et al. (2009). At $\theta=\theta_{\text {clean }}$, there is a strong decrease of the flow rate (strong increase of local vorticity) near the bubble surface induced by the change of interface condition from "zero tangential stress" to "no-slip condition". This flow rate decrease results in a dilatation of the streamlines. The same effect is observed for the particles trajectory, since the inertial free particles follow the streamlines. It put the particle trajectory far away from the bubble surface. For $\theta_{\text {clean }} \geq 90^{\circ}$, since the contact point is always located at the forward part of the bubble surface, there are little influence of $\theta_{\text {clean }}$ on the evolution of $\theta_{c}$, which is very close to that observed for a clean bubble. Meanwhile for $\theta_{\text {clean }}<90^{\circ}$, two situations are observed and can be distinguished by a critical angle $\theta_{\text {crit }}$ for the surface contamination level:

- If $\theta_{\text {clean }}>\theta_{\text {crit }}$, flow rate on the clean part (controlled by the tangential velocity) is not affected by the interface contamination and is always larger than that near the contaminated surface (controlled by the velocity gradient). Consequently, the collision occurs on the mobile part of the bubble surface and $\theta_{c}$ can not be larger than $\theta_{\text {clean }}$.

- If $\theta_{\text {clean }}<\theta_{\text {crit }}$, the velocity gradient reaches locally a maximum close to the value obtained for a fully contaminated bubble. Consequently, particles-bubble collision is then independent 
on the surface contamination level and $E_{\text {coll }}$ and $\theta_{c}$ are those observed for a fully contaminated bubble.

The value of the critical angle $\theta_{\text {crit }}$ has been shown to be related to bubble's Reynolds number (Legendre et al., 2009):

$\theta_{\text {crit }}=33^{\circ} \frac{R e_{b}+4.4}{R e_{b}+2.5}$

For example at $R e_{b}=1, \theta_{\text {crit }}=50^{\circ}$. Therefore, for all $\theta_{\text {clean }}<50^{\circ}$, the contact point of the grazing trajectory may be located on the immobile part of the bubble surface, depending on the particle size. By using the relationship given by Legendre et al. (2009):

$\frac{r_{p}}{r_{b}}=\frac{f^{m}}{f^{i m}} \sin ^{n} \frac{\theta_{\text {crit }}}{n} \sin \frac{3 \theta_{\text {crit }}}{4}$

with

$f^{m}=\frac{16+3.315 R e_{b}^{0.5}+3 R e_{b}}{16+3.315 R e_{b}^{0.5}+R e}, \quad f^{i m}=1+\frac{3 / 16 R e_{b}}{1+0.249 R e_{b}^{0.56}}, \quad n=\frac{2+0.2 R e_{b}^{0.5}}{1+0.2 R e_{b}^{0.5}}$

we find $\theta_{\text {crit }} \approx 20^{\circ}$ for the inertial free particle with $r_{p} / r_{b}=0.01$, which agrees with the results shown in Fig. 16b: for bubbles with $\theta_{\text {clean }}=20^{\circ}$, the collision happens near the equator with $\theta_{c}=83^{\circ}$; while for bubbles with $\theta_{\text {clean }}>20^{\circ}$, the collisions angles are limited on the mobile part and closed to $\theta_{\text {clean }}$.

Meanwhile, as $S t_{p}$ increases, the evolution of the collision angle becomes more complex. Considering the large Stokes number $S t_{p}=0.1$, whatever the contamination level of the interface, the collision angle is under $45^{\circ}$, except for totally contaminated interface. So the collision is rather governed by the inertial forces. In other words, the particle inertia is great enough to push the particle to go straightly toward the bubble and deposit on the front part of the bubble surface. Despite particle inertia force, the collision angle is always limited by $\theta_{\text {clean }}$. It is interesting to note an abrupt change of collision angle for $R_{b}=1$ and $\theta_{\text {clean }}=20$ (see Fig. 16b, symbol " $\triangle$ " ). To well understand this behavior, critical particle trajectories are reported in Fig. 17, for different $S t_{p}$ varying from 0.002 to 0.5 . Here we use the polar coordinate $(r, \theta)$. The dash line represents the location of the bubble's surface.

For small $S t_{p}$, particle grazing trajectories reveal that there are two minimal distances between the bubble and the particle. Indeed since the inertial effect is not important for such particles, they approaches to the front part of bubble surface as observed for the case of a clean bubble. Near $\theta_{\text {clean }}\left(=20^{\circ}\right.$ in Fig. 17), the jump of the streamlines caused by the change of interface condition ("slip" to "no-slip") pushes the particle away from the interface. On the equatorial part of the bubble, streamlines are once again put closer to the interface when the strong increase of vorticity has been evacuated and the flow has accelerated. Therefore, particles that have not been captured by the front part can be trapped near the equatorial part (Sarrot et al., 2005). When $S t_{p}$ becomes important $(\geq 0.2)$, collision is rather controlled by inertial deposition at the front part of the bubble and $\theta_{c}$ is then controlled by $\theta_{\text {clean }}$ as explained before. However, this phenomenon characterized by two minimum distances can only be observed if $\theta_{\text {clean }}<\theta_{\text {crit }}$.

\subsubsection{Effect the gravitational forces $\left(S t_{p} \neq 0\right.$ and $u_{s} \neq 0$ )}

In this section we consider all the effects induced by inertia. Fig. 18a and b shows, respectively, the evolution of the collision efficiency and collision angle as function of Stokes number $S t_{p}$. Different levels of contamination are reported for bubble Reynolds number $R e_{b}=100$ and a particle to bubble size ratio $r_{p} / r_{b}=0.01$. The contamination angle $\theta_{\text {clean }}$ varies from $\theta_{\text {clean }}=0^{\circ}$ (fully contaminated bubble) to $\theta_{\text {clean }}=180^{\circ}$ (clean bubble).

Notably, when the gravitation forces are considered, the collision efficiency is significantly increased, compared to the results presented in the previous section. This figure reveals again two different regimes separated by $S t_{p}^{\text {th }} \sim 0.03$. The effect of the interface contamination is only observed for $S t_{p} \leq S t_{p}^{\text {th }}$. As observed in the previous section, $E_{\text {coll }}^{\text {im }}<E_{\text {coll }}\left(\theta_{\text {clean }}\right)<E_{\text {coll }}^{m}$, as $\theta_{\text {clean }}$ varies from $20^{\circ}$ to $90^{\circ}$. Meanwhile, for $S t_{p} \geq S t_{p}^{\text {th }}$ (at $r_{p} / r_{b}=0.01$ ), $E_{\text {coll }}$ increases monotonously with $S t_{p}$ and is independent of $\theta_{\text {clean }}$. Considering the collision angle $\theta_{c}$, three different behaviors are observed:

- $\theta_{\text {clean }} \leq \theta_{\text {crit }}$ : Collision behavior is the same as that of a fully contaminated bubble and governed by the "positive inertial effect" and gravitational forces. For $R e_{b}=100$ and $r_{p} / r_{b}=0.01$, one has a critical angle $\theta_{\text {crit }}=10^{\circ}$.

- $\theta_{\text {crit }}<\theta_{\text {clean }}<90^{\circ}$ : Collision angle $\theta_{c}$ is observed to experience three stages evolution as described by Fig. 19, for example, $\theta_{\text {clean }}=45^{\circ}$. In this figure, critical particles trajectories are plotted for the particles of size ratio $r_{p} / r_{b}=0.01$ and the Stokes number varying between 0.002 and 1.0. The dash line represents the location of the bubble's surface. Before focussing on the contact point at the interface, we can first note that at $r / r_{b}=0.1$, the distance between the particle's trajectory and the symmetry axis $(\theta=0)$ is significantly widened with the increase of the Stokes number. It also indicates the increase of collision efficiency, since $E_{\text {coll }} \sim\left(r_{p} / r_{b}\right)^{2}$.

For small Stokes number $\left(S t_{p} \leq 0.01\right)$, particle bubble collision only occurs on the mobile part of the interface

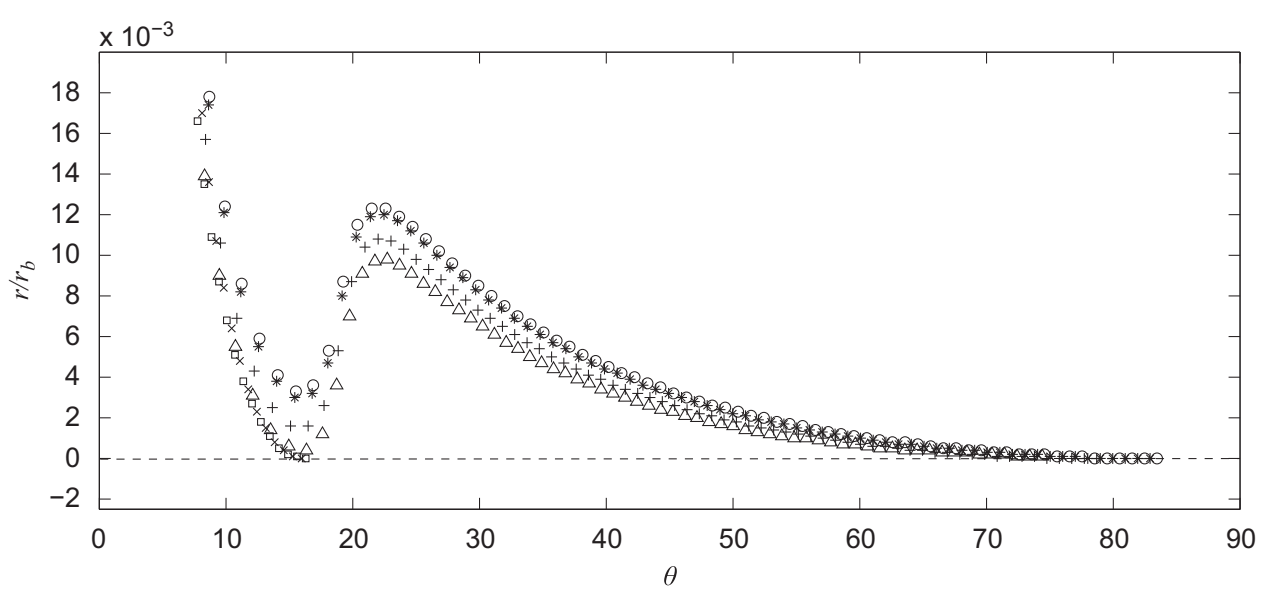

Fig. 17. Particles grazing trajectories in the vicinity of a partially contaminated bubble for $\theta_{\text {clean }}=20^{\circ}\left(R e_{b}=1, r_{p} / r_{b}=0.01\right)$.,$S t_{p}=0.002 ; *, S t_{p}=0.01 ;+, S t_{p}=0.02 ; \triangle$, $S t_{p}=0.1 ; \square, S t_{p}=0.2 ; \times, S t_{p}=0.5,--$, bubble surface. 

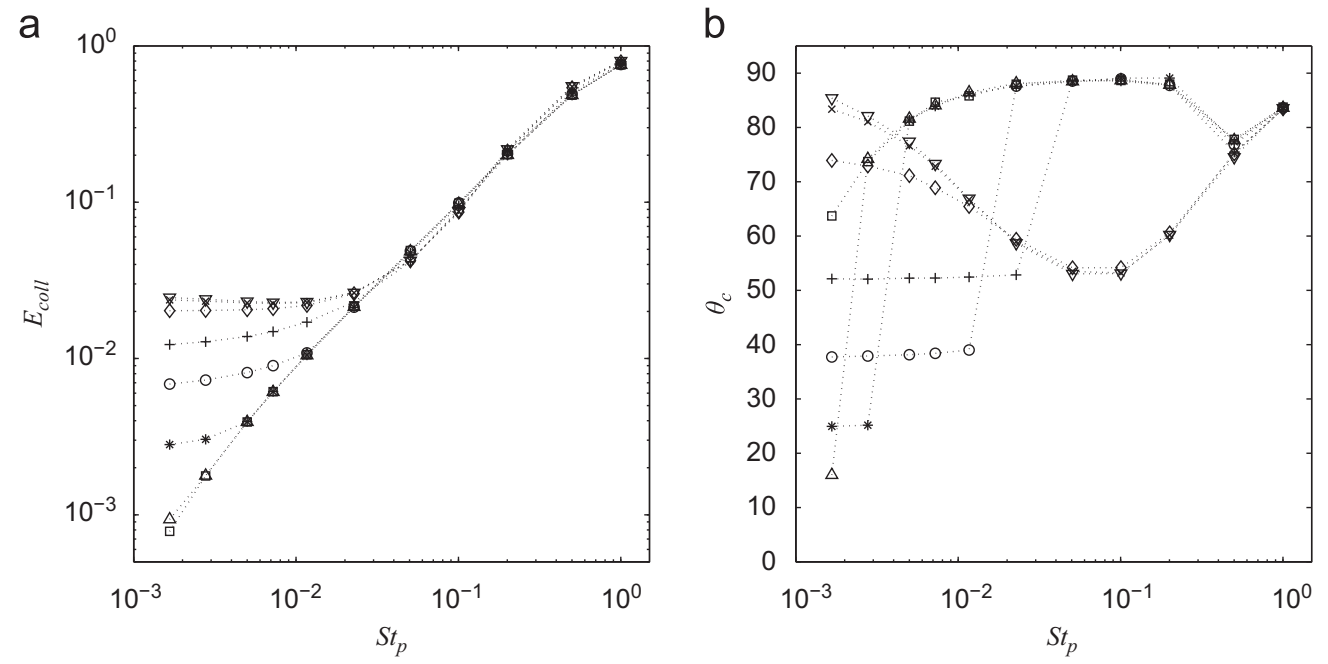

Fig. 18. Collision efficiency $\left(E_{\text {coll }}\right)$ and collision angle $\left(\theta_{c}\right)$ vs. Stokes number $\left(S t_{p}\right)$ for a partially contaminated bubble $\left(R e_{b}=100\right.$ and $\left.r_{p} / r_{b}=0.01\right)$. $\square, \theta_{c l e a n}=00^{\circ} ; \triangle$, $\theta_{\text {clean }}=20^{\circ} ; *, \theta_{\text {clean }}=30^{\circ} ; \circ, \theta_{\text {clean }}=45^{\circ} ;+, \theta_{\text {clean }}=60^{\circ} ; \diamond, \theta_{\text {clean }}=90^{\circ} ; \times, \theta_{\text {clean }}=120^{\circ} ; \nabla, \theta_{\text {clean }}=180^{\circ}$. (a) Collision efficiency. (b) Collision angle.

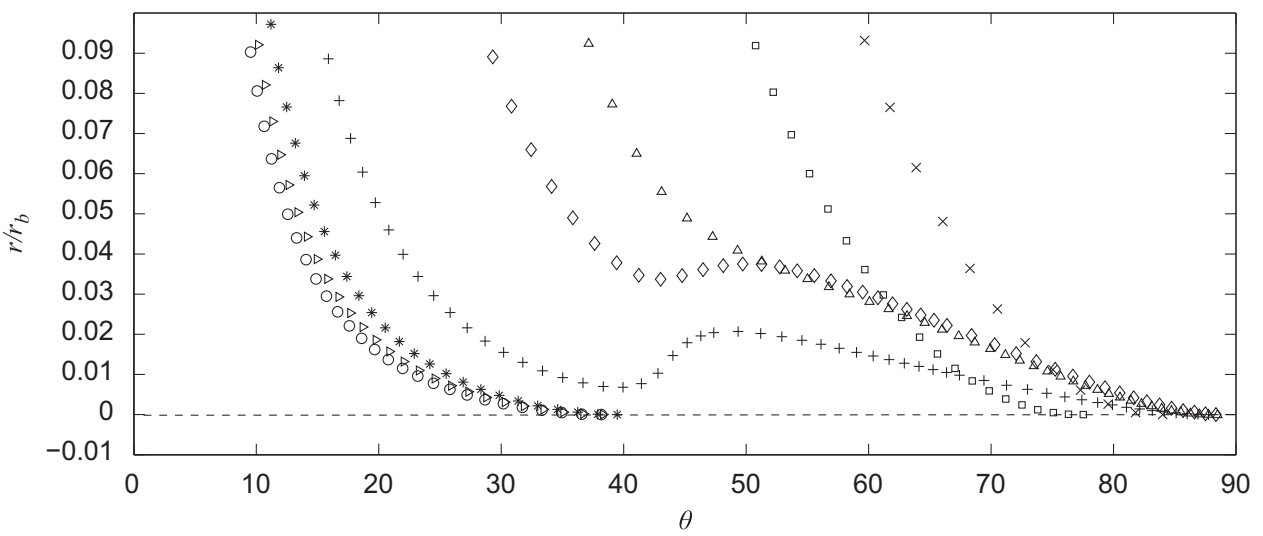

Fig. 19. Particles $\left(r_{p} / r_{b}=0.01\right)$ grazing trajectories in the vicinity of a partially contaminated bubble for $\theta_{\text {clean }}=45^{\circ}$ and $R e_{b}=100$. $\circ, S t_{p}=0.002 ; \triangleright, S t_{p}=0.005 ; *$, $S t_{p}=0.01 ;+, S t_{p}=0.02 ; \diamond, S t_{p}=0.1 ; \triangle, S t_{p}=0.2 ; \square, S t_{p}=0.5 ; \times, S t_{p}=1.0 ;---$, bubble surface.

and collision angle is limited by the surface contamination level $\left(\theta_{c}<\theta_{\text {clean }}\right)$.

For large Stokes number $\left(S t_{p} \leq 0.2\right)$, the effect of sedimentation and particle inertia permit the particle to overcome the jump of streamlines induces by the change of interface condition. As observed for $S t_{p}=0.5$ and $S t_{p}=1$ in Fig. 19, it makes the particles easier to collide with the bubble surface at the location of the first minimal distance to the interface and the collision occurs on the contaminated part.

For intermediate Stokes $\left(0.01<S t_{p}<0.20\right)$, the trajectories near the surface are influenced by the deviation of the streamlines near $\theta_{\text {clean }}$. The jump of streamlines makes the collision near $\theta_{\text {clean }}$ to be impossible and particles can only approach to the interface at the location of the second minimal distance on the immobile part. For such a situation, the collision angle can become larger than the collision angle observed for larger Stokes number as shown in Fig. 18b.

- $\theta_{\text {clean }} \geq 90^{\circ}$ : Unlike other partially contaminated bubble, the collision angle evolves gradually and there is not a sudden jump of $\theta_{c}$, as it is shown in Fig. 18b. For a bubble with $\theta_{\text {clean }}=90^{\circ}$, the jump of the streamline locates near the $90^{\circ}$, so the effect of "two minimal distances" can never happen. $\theta_{c}$ is first reduced by the negative effect of "centrifuge forces" and then increased under the influence of the combined effect of gravity and inertial forces. It can be also noted that for $\theta_{\text {clean }} \geq 120^{\circ}$, the evolution of the collision angle with $S t_{p}$ is the same to that of a clean bubble.

\subsection{Modeling}

The effect of bubble contamination has been presented in the previous section by varying the bubbles Reynolds number $\left(1 \leq \operatorname{Re}_{b} \leq 100\right)$, the radius ratio $\left(0.001 \leq r_{p} / r_{b} \leq 0.02\right)$ and the particle Stokes number $\left(0.001 \leq S t_{p} \leq 1\right)$. The objective of this section is to propose a simple model that permits to describe the collision efficiency for a partially contaminated surface. In practice the level of bubble contamination can be deduced from the bubble size and terminal velocity (Huang et al., 2011). A detailed inspection of the numerical results replaces that a simple rough estimation of the collision efficiency can be proposed as follow:

$E_{\text {coll }}\left(R e_{b}, S t_{p}, \theta_{\text {clean }}\right)=E_{\text {coll }}^{0}\left(R e_{b}, \theta_{\text {clean }}\right)+K \frac{S t_{p}^{n}}{\left(1+S t_{p}\right)^{n}}$

The first term $E_{0}\left(R e_{b}, \theta_{\text {clean }}\right)$ in Eq. (37) represents the collision efficiency for an inertial-free particle $S t_{p}=0$. The model of Legendre et al. (2009) can be used to describe the effect of the level of contamination on $E_{\text {coll }}$.

$E_{\text {coll }}^{0}=2 \sin \theta_{c} \frac{r_{p}}{r_{b}}\left(1+\frac{r_{p}}{r_{b}}\right) \frac{u_{\Gamma}}{u_{b}}$ 

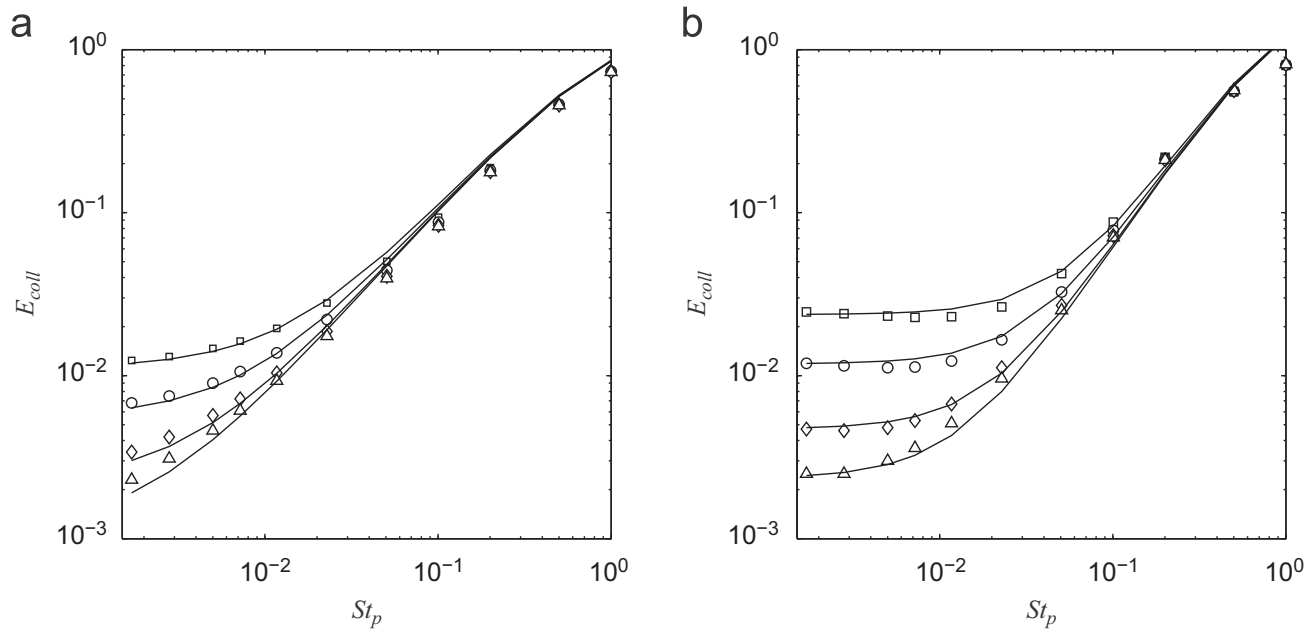

Fig. 20. Collision efficiency for a clean bubble. DNS results $\square, r_{p} / r_{b}=0.01 ; \circ, r_{p} / r_{b}=0.005 ; \diamond, r_{p} / r_{b}=0.002 ; \triangle, r_{p} / r_{b}=0.001 ;-$, Eq. (37), with $K=2.5$ and $n=1.5$ for $R e_{b}=100, K=2.5$ and $n=1.2$ for $R e_{b}=1$. (a) $R e_{b}=100$. (b) $R e_{b}=1$.
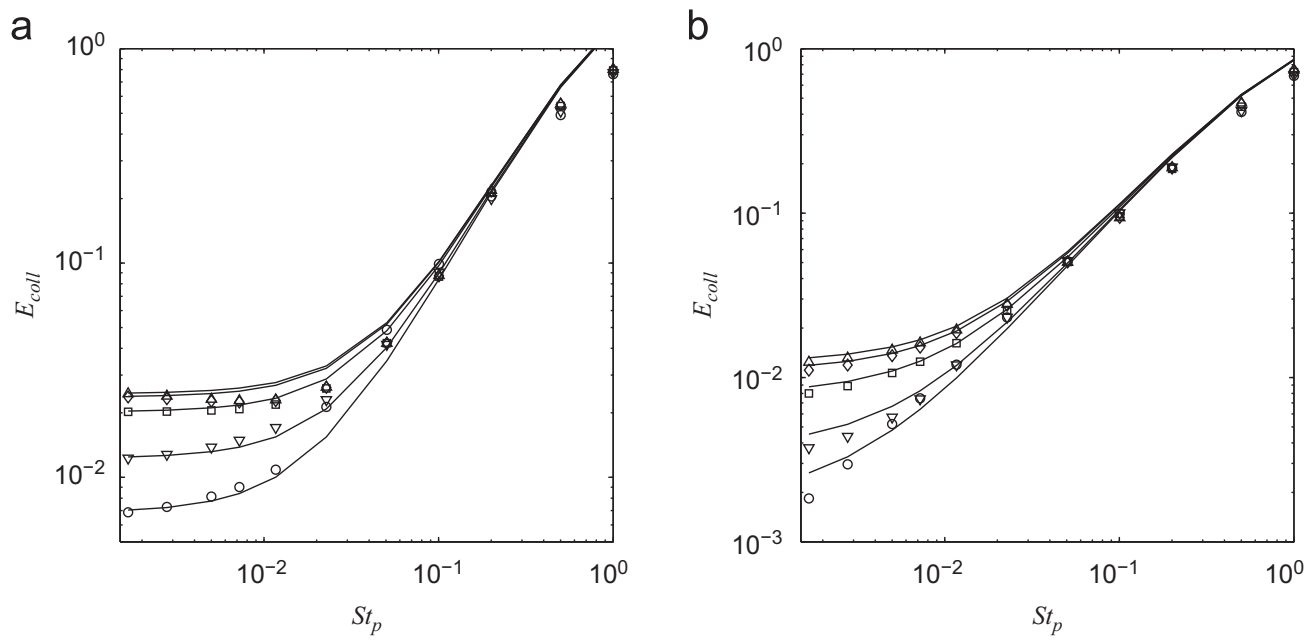

Fig. 21. Collision efficiency for a partially contaminated bubble for $r_{p} / r_{b}=100$. DNS results $\triangle, \theta_{\text {cap }}=180^{\circ} ; \diamond, \theta_{\text {cap }}=120^{\circ} ; \square, \theta_{\text {cap }}=90^{\circ} ; \circ, \theta_{\text {cap }}=45^{\circ} ;-$, Eq. (37), with $K=2.5$ and $n=1.5$ for $R e_{b}=100, K=2.5$ and $n=1.2$ for $R e_{b}=1$. (a) $R e_{b}=100$. (b) $R e_{b}=1$.

where $u_{\Gamma}$ is the maximum tangential velocity at the bubble surface given by

$u_{\Gamma}=\frac{u_{b}}{2} f^{180}\left(\operatorname{Re}_{b}\right) \sin ^{n}\left(\frac{\theta_{\text {clean }}}{n}\right) \quad$ if $\theta_{\text {clean }} \leq \frac{k \pi}{2}$

$u_{\Gamma}=\frac{u_{b}}{2} f^{180}\left(\operatorname{Re}_{b}\right)$ if $\theta_{\text {clean }}>\frac{k \pi}{2}$

with $k=\left(2+0.2 R e_{b}^{0.5}\right) /\left(1+0.2 R e_{b}^{0.5}\right)$ and $\theta_{c}$ is the collision angle defined by the grazing trajectory:

$\theta_{c}=\frac{2+0.16 R e_{b}^{0.5}}{3+0.16 \operatorname{Re}_{b}^{0.5}} \theta_{\text {clean }} \quad$ if $0<\theta_{\text {clean }} \leq \frac{\pi}{2}$

$\theta_{c}=\frac{3}{4} \theta_{\text {clean }} \quad$ if $\frac{\pi}{2}<\theta_{\text {clean }}<\frac{2 \pi}{3}$

$\theta_{c}=\arccos \left[\left(\sqrt{X^{2}+3 Y^{2}}-X\right) / 3 Y\right]$ if $\frac{2 \pi}{3} \leq \theta_{\text {clean }}<\pi$

where $X$ and $Y$ are the same as in Eq. (32) given by Nguyen (1998).

The second term in Eq. (37) represents the contribution of the inertial forces. We chose to use Schulze model (Eq. (34)) by modifying the coefficients $K$ and $n$. These two coefficients are slightly dependent on the Reynolds number. For $\operatorname{Re}_{b}=100, K \sim 2.5$ and $n=1.5$, while for $R e_{b}=1, K \sim 1.7$ and $n=1.2$.

The model given by expression (37) is compared to the numerical simulations in Fig. 20 for a clean bubble and in Fig. 21 for a partial contaminated bubble $\left(\theta_{\text {clean }}=120^{\circ}, \theta_{\text {clean }}=90^{\circ}, \theta_{\text {clean }}=45^{\circ}\right.$ ). It can be noted that this model gives a rather good accordance with the simulations. For $R_{b}=1$, a small discrepancy is observed for the low $r_{p} / r_{b}$. This discrepancy may be due to the gravitational effect not integrated in relation (37). The importance of the gravitational effect at small $S t_{p}$ and small $r_{p} / r_{b}$ can clearly be seen in Fig. 10, even if this figure has been plotted for $R_{b}=100$. The prediction at high level of contamination $\left(\theta_{\text {clean }} \leq 30^{\circ}\right.$ not reported in the figure) is not satisfactory with this model. The coefficient $K$ and $n$ becomes more complex because of their dependency with the parameters of the problem, i.e. bubble's Reynolds number.

\section{Conclusions}

In this paper, the inertial particle bubble collision efficiency has been evaluated by solving the full particle trajectory equation of particles moving in the flow field generated by a rising bubble. Direct numerical simulations have been performed for 
$1<R e_{b}<100$ by taking into consideration the interface contamination level via the stagnant cap model. The effect of inertia on the collision efficiency has been examined at first. It was found that $E_{\text {coll }}$ is strongly influenced by inertial forces. For all the cases considered, particle inertia has a positive effect at large $S t_{p}$, which results in a sharp increase in the value of $E_{\text {coll }}$. On the contrary, at small $S t_{p}$, a negative effect has been observed when the particle moves near the bubble equator, where the tangential component of the surface velocity reaches its maximum for clean or nearly clean bubbles. This tangential velocity creates a "centrifugal force" that pulls particles away from the interface and makes collision impossible above a certain angle. As a result, $E_{\text {coll }}$ decreases as $S t_{p}$ increases. For a partially contaminated bubble $\left(\theta_{\text {clean }}<90^{\circ}\right)$, the flow field around the bubble is modified and the collision efficiency depends strongly on $\theta_{\text {clean }}$. If $\theta_{\text {clean }}>\theta_{\text {crit }}$, the contact point of the grazing trajectory can only be situated on the mobile interface, as the surface velocity decreases with $\theta_{\text {clean }}$, the negative effect being greatly reduced. If $\theta_{\text {clean }}<\theta_{\text {crit }}$, the contact point may be on both mobile and immobile part of the interface. $E_{\text {coll }}$ behaves as that of a fully contaminated bubble and only the positive inertial effect is observed. The critical angle $\theta_{\text {crit }}$ depends on the bubble's Reynolds number. Second, the influence of the gravitational settling on the collision behavior has been analyzed. Its contribution to the collision efficiency becomes important, when $u_{s}>0.01$, which should not be neglected. Finally a simple model has been proposed that makes possible to describe collision efficiency for any clean or contaminated bubble, inertial or non-inertial particles, but not yet for situations dominated by the gravitational sedimentation.

\section{Nomenclature}

\section{Roman symbols}

\begin{tabular}{|c|c|}
\hline$C_{D}$ & drag coefficient $(-)$ \\
\hline$C_{M}$ & added mass coefficient $(-)$ \\
\hline$C_{L}$ & lift coefficient $(-)$ \\
\hline$E_{\text {coll }}$ & collision efficiency $(-)$ \\
\hline$F_{R}$ & electrostatic force $(\mathrm{N})$ \\
\hline$F_{A}$ & Van de Waals forces $(\mathrm{N})$ \\
\hline$F_{H}$ & hydrophobic force $(\mathrm{N})$ \\
\hline$g$ & gravitational constant $\left(\mathrm{m} \mathrm{s}^{-1}\right)$ \\
\hline $\operatorname{Re}$ & Reynolds number $(-)$ \\
\hline$r$ & radius $(\mathrm{m})$ \\
\hline$r_{c}$ & radius of grazing trajectory $(\mathrm{m})$ \\
\hline St & Stokes number (-) \\
\hline$S t^{t h}$ & critical Stokes number $(-)$ \\
\hline$u$ & normalized velocity of liquid $(-)$ \\
\hline$U$ & liquid velocity $\left(\mathrm{m} \mathrm{s}^{-1}\right)$ \\
\hline$u_{s}$ & normalized settling velocity $(-)$ \\
\hline$U_{s}$ & velocity of sedimentation $\left(\mathrm{m} \mathrm{s}^{-1}\right)$ \\
\hline$v$ & normalized velocity of particle $(-)$ \\
\hline$V$ & particle velocity $\left(\mathrm{m} \mathrm{s}^{-1}\right)$ \\
\hline
\end{tabular}

Greek symbols

$\mu$
$\theta$
$\theta_{c}$
$\theta_{\text {clean }}$
$\theta_{\text {crit }}$
$\rho$
$\hat{\rho}$
$\tau$
$\Psi$

dynamic viscosity (Pa s)

polar angle $\left({ }^{\circ}\right)$

collision angle $\left({ }^{\circ}\right)$

clean angle $\left({ }^{\circ}\right)$

critical angle $\left({ }^{\circ}\right)$

density $\left(\mathrm{kg} \mathrm{m}^{-3}\right)$

particle to liquid density ratio $\left(\mathrm{kg} \mathrm{m}^{-3}\right)$

characteristic time (s)

grazing trajectory $(-)$

\section{Subscripts}

$\begin{array}{ll}b & \text { bubble } \\ l & \text { liquid } \\ p & \text { particle }\end{array}$

Superscripts

im immobile interface

$m \quad$ mobile interface

\section{Acknowledgments}

The authors would like to especially thank the CNRS Federation FERMAT for the financial and technical support.

\section{References}

Alves, S.S., Orvalho, S.P., Vasconcelos, J.M.T., 2005. Effect of bubble contamination on rise velocity and mass transfer. Chemical Engineering Science 60, 1-9.

Bel Fdhila, R., Duineveld, P.C., 1996. The effect of surfactant on the rise of a spherical bubble at high Reynolds and Peclet number. Physics Fluids 8, 310.

Climent, E., Magnaudet, J., 1999. Large-scale simulations of bubble-induced convection in a liquid layer. Physics Review Letters 82, 4827.

Craig, V.S.J., Ninham, B.W., Pashley, R.M., 1999. Direct measurement of hydrophobic forces a study of dissolved gas, approach rate, and neutron irradiation. Langmuir 15 (4), 1562-1569.

Cuenot, B., Magnaudet, J., Spennato, B., 1997. The effects of slightly soluble surfactants on the flow around a spherical bubble. Journal of Fluid Mechanics 339 (25-53).

Dai, Z., Dukhin, S., Fornasiero, D., Ralston, J., 1998. The inertial hydrodynamic interaction of particles and rising bubbles with mobile surface. Journal of Colloid and Interface Science 197, 275-292.

Dai, Z., Fornasiero, D., Ralston, J., 2000. Particle-bubble collision models-a review. Advances in Colloid and Interface Science 85, 231-256.

Dobby, G.S., Finch, J.A., 1987. Particle size dependence in flotation derived from a fundamental model of the capture process. International Journal of Mineral Processing 21, 241-251.

Dukhin, S.S., 1983. Critical value of the Stokes number and the Sutherland formula. Kolloidn Zhurnal 45 (2), 207-218.

Dukhin, S.S., Kretzschmar, G., Miller, R., 1995. Dynamic of Adsorption at Liquid Interface. Elsevier, Amsterdam.

Figueroa-Espinoza, B., Legendre, D., Zenit, R., 2008. The effect of confinement on the motion of a single clean bubble. Journal of Fluid Mechanics 616, 419-443.

Flint, L.R., Howerth, W.J., 1971. The collision efficiency of small particles with spherical air bubbles. Chemical Engineering Science 26, 1155-1168.

Gaudin, A.M., 1957. Flotation, second ed. McGraw Hill Book Co. Inc., New York.

Hamaker, H., 1937. Physica 4, 1058.

Happel, J., Brenner, H., 1965. Low Reynolds Number Hydrodynamics with Special Applications to Particulate Media. Prentice-Hall Englewood Cliffs, New York

Heindel, T.J., Bloom, F., 1999. Exact and approximate expressions for bubbleparticle collision. Journal of Colloid and Interface Science 213 (1), 101-111.

Huang, Z., Legendre, D., Guiraud, P., 2011. A new experimental method for determining particle capture efficiency in flotation. Chemical Engineering Science 66, 982-997.

Kim, S., Karilla, S.J., 1991. Microhydrodynamics: Principles and Selected Applications. Butterworth-Heinemann, Boston, MA.

Legendre, D., Magnaudet, J., 1998. The lift force on a spherical bubble in a viscous linear shear flow. Journal of Fluid Mechanics 368, 81-126.

Legendre, D., Magnaudet, J., Mougin, G., 2003. Hydrodynamic interactions between two spherical bubbles rising side by side in a viscous liquid. Journal of Fluid Mechanics 497, 133-166.

Legendre, D., Sarrot, V., Guiraud, P., 2009. On the particle inertia-free collision with a partially contaminated spherical bubble. International Journal of Multiphase Flow 35, 163-170.

Magnaudet, J., Rivero, M., Fabre, J., 1995. Accelerated flows past a rigid sphere or a spherical bubble. Part1 steady straining flow. Journal of Fluid Mechanics 284, 97-135.

Maxey, M.R., Riley, J.J., 1983. Equation of motion for a small rigid sphere in a nonuniform flow. Physics Fluids 24 (4), 883-889.

McLaughlin, J.B., 1991. Initial migration of a small sphere in linear shear flows. Journal of Fluid Mechanics 244, 261.

McLaughlin, J.B., 1996. Numerical simulation of bubble motion in water. Journal of Colloid Interface Science 184, 614-625.

Merle, A., Legendre, D., Magnaudet, J., 2005. Forces on a high-Re spherical bubble in a turbulent flow. Journal of Fluid Mechanics 532, 53-62.

Mishchuk, N., 2005. The role of hydrophobicity and dissolved gases in nonequilibrium surface phenomena. Colloids and Surfaces A: Physicochemical Engineering Aspects 267, 139-152. 
Mishchuk, N., Ralston, J., Fornaserio, D., 2002. Influence of dissolved gas on the Van der Waals forces between bubbles and particles. The Journal of Physical Chemistry A 106, 689-696.

Mishchuk, N.A., Koopal, L.K., Dukhin, S.S., 2001. Microflotation suppression and enhancement caused by particle/bubble electrostatic interaction. Journal of Colloid and Interface Science 237, 208-223.

Moruzzi, R.B., Reali, M.A.P., 2010. Characterization of micro-bubble size distribution and flow configuration in DAF contact zone by a non-intrusive image analysis system and tracer tests. Water Science and Technology 61 (1) 253-262.

Nguyen, A.V., 1994. The collision between fine particles and single air bubbles in flotation. Journal of Colloid and Interface 162, 123-128.

Nguyen, A.V., 1998. Particle-bubble encounter probability with mobile bubble surface. International Journal of Mineral Processing 55, 73-86.

Nguyen, A.V., 1999. Hydrodynamics of liquid flows around air bubbles in flotation a review. International Journal of Mineral Processing 56, 165-205.

Nguyen, A.V., 2003. New method and equations for determining attachment tenacity and particle size limit in flotation. International Journal of Minera Processing 68, 167-182.

Nguyen, A.V., Evans, G.M., 2004. Movement of fine particles on an air bubble surface studied using high-speed video microscopy. Journal of Colloid and Interface Science 273, 271-277.

Nguyen, A.V., Kmet, S., 1992. Collision efficiency for fine mineral particles with single bubble in a counter current flow regime. International Journal of Mineral Processing 35, 205-223.

Nguyen, A.V., Schulze, H.J., 2004. Colloidal Science of Flotation. Marcel Dekker, New York, USA.

Nguyen, A.V., Ralston, J., Schulze, H.J., 1998. On modelling of bubble particle attachment probability in flotation. International Journal of Mineral Processing 53 (4), 225-249.

Nguyen, A.V., Evans, G.M., Schulze, H.J., 2001. Prediction of Van der Waals interaction in bubble particle attachment in flotation. International Journal of Mineral Processing 61, 155-169.

Nguyen, C.M., Nguyen, A.V., Miller, J.D., 2006. Computational validation of the generalized Sutherland equation for bubble-particle encounter efficiency in flotation. International Journal of Mineral Processing 81 (3), 141-148.

Nguyen, P.T., Nguyen, A.V., 2009. Validation of the generalised Sutherland equation for bubble particle encounter efficiency in flotation: Effect of particle density. Minerals Engineering 22, 176-181.

Phan, C.M., Nguyen, A.V., Miller, J.D., Evansa, G.M., Jameson, G.J., 2003. Investigations of bubble particle interactions. International Journal of Mineral Processing 72, 239-254

Plate, H., 1989. Modellierung des Flotationsprozeses auf der Grundlage der Mikroprozess. Ph.D. Thesis, AdW der DDR, FIA Freiberg.
Ralston, J., Dukhin, S.S., Mishchuk, N.A., 1999. Inertial hydrodynamic particlebubble interaction in flotation. International Journal of Mineral Processing 56 (a), 207-256.

Ralston, J., Dukhin, S.S., Mishchuk, N.A., 2002. Wetting film stability and flotation kinetics. Advances in Colloid and Interface Science 95, 145-256.

Sadhal, S.S., Johnson, R.E., 1983. Stokes flow past bubbles and drops partially coated with thin films. Journal of Fluid Mechanics 126, 237-247.

Sam, A., Gomez, C.O., Finch, J., 1996. Axial velocity profiles of single bubbles in water/frother solution. International Journal of Mineral Processing 47, 177-196.

Sarrot, V., Guiraud, P., Legendre, D., 2005. Determination of the collision frequency between bubbles and particles inflotation. Chemical Engineering Science 60 , 6107-6117.

Savic, P., 1953. Circulation and distortion of liquid drops falling through a viscous medium. Technical Report MT-22. National Research Council Canada, Division Mechanical Engineering.

Schiller, L, Nauman, A, 1935. A drag coefficient correlation. V.D.I. Zeitung 77, 318

Schulze, H.J., 1989. Hydrodynamics of bubble-mineral particle collisions. Mineral Processing and Extractive Metal Review 5, 43-76.

Stone, H.A., 1990. A simple derivation of the time-dependent convective-diffusion equation for surfactant transport along a deforming interface. Physics Fluids A 2, 111-112.

Sutherland, K.L., 1948. Physical chemistry of flotation XI. Kinetics of the flotation process. Journal of Physical Chemistry 52, 394-425.

Usui, S., Barouch, E., 1990. Effect of adsorbed layers on the Van der Waals interaction between particles and bubbles in aqueous media. Journal of Colloid and Interface Science 137 (1), 281-288.

Vold, M.J., 1961. The effect of adsorption on the Van der Waals interaction of spherical colloidal particles. Journal of Colloid and Interface Science 16, 1.

Weber, M.E., 1981. Collision efficiencies for small particles with a spherica collector at intermediate Reynolds numbers. Journal of Separation Process Technology 2 (1), 29-33.

Weber, M.E., Paddock, D., 1983. Interceptional and gravitational collision efficiencies for single collectors at intermediate Reynolds numbers. Journal of Colloid Interface Science 94 (2), 328-335.

Yoon, R.H., 2000. The role of hydrodynamic and surface forces in bubble-particle interaction. International Journal of Mineral Processing 58, 129-143.

Yoon, R.H., Luttrell, G.H., 1989. The effect of bubble size on fine particle flotation. Mineral Processing and Extractive Metal Review 5, 101-122.

Yordan, J.L., Yoon, R.H., 1989. Induction time measurement for the quartz amine flotation system. In: 115th SME ANN Meeting. Littleton Co., p. 10.

Zholkovskij, E.K., Koval'chuk, V.I., Dukhin, S.S., Miller, R., 2000. Dynamics of rear stagnant cap formation at low Reynolds numbers: 1 slow sorption kinetics. Journal of Colloid and Interface Science 226, 51-59. 